\title{
Welfare Egalitarianism with Other-Regarding \\ Preferences
}

by

Rafael Treibich

Discussion Papers on Business and Economics

No. 22/2014

FURTHER INFORMATION

Department of Business and Economics

Faculty of Business and Social Sciences University of Southern Denmark

Campusvej 55

DK-5230 Odense M

Denmark

Tel.: +456550 3271

Fax: +456550 3237

E-mail: 1ho@sam.sdu.dk

http://www.sdu.dk/ivoe 


\title{
WELFARE EGALITARIANISM WITH OTHER-REGARDING PREFERENCES
}

\author{
Rafael Treibich* \\ University of Southern Denmark ${ }^{\dagger}$
}

\begin{abstract}
We study the fair allocation of a one-dimensional and perfectly divisible good when individuals have other-regarding preferences. Assuming no legitimate claims and purely ordinal preferences, how should society measure social welfare so as to satisfy basic principles of efficiency and fairness? We define the egalitarian equivalent as the size of the egalitarian allocation which leaves the individual indifferent to the current allocation. In two simple models of average and positional externalities, we characterize the class of social preferences which give full priority to the individual with the lowest egalitarian equivalent in the economy.
\end{abstract}

Keywords: Fair Allocation, Social Welfare, Externalities, Behavioral Economics.

JEL Classification Numbers: D62, D63, D64, D71.

\section{INTRODUCTION}

We consider the problem of allocating a one-dimensional and perfectly divisible good when preferences exhibit consumption externalities. Individuals care both about how much they receive and how much the others receive, which means their preferences are defined over complete allocations. In contrast with the existing literature, mostly inspired by the Theory of Fair Allocation (Thomson 2010 [41]), we follow the recent approach of Fair Social Choice (Fleurbaey and Maniquet 2011 [17]). Our objective is to construct complete orderings of all allocations, using as only information the profile of ordinal preferences in the economy. Defining such a comprehensive measure of social welfare is particularly important when the subset of feasible allocations is limited or uncertain. This may happen in particular if the social planner has to respect various types of legal, political, technological or informational constraints. Our objective here is to construct social ordering functions which satisfy appealing principles of efficiency and fairness.

Despite an extensive literature on other-regarding preferences (Frank 1985 [21], Sobel 2005 [40], Clark et al. 2008 [5]), and a wide array of applications (such as growth ${ }^{3}$, optimal taxation ${ }^{4}$ or general

${ }^{*} \operatorname{rtr}[\mathrm{at}] \mathrm{sam} . \mathrm{sdu} . \mathrm{dk}$

This version: November $20^{\text {th }} 2014$.

I am grateful to Marc Fleurbaey and Jean-François Laslier for their very helpful advice and suggestions. I would also like to thank Yukio Koriyama, Antonin Macé, François Maniquet, Eduardo Perez, Paolo Piacquadio, Yves Sprumont, Karol Szwagrzak, Giacomo Valletta and seminar participants in Lisbon, Louvain-la-Neuve, Maastricht, Marseille, Montréal, Paris and Odense.

${ }^{3}$ Corneo and Jeanne 1997 [6], Cooper et al. 2001 [7].

${ }^{4}$ Boskin and Sheshinski 1978 [3], Persson 1995 [35], Ireland 2001 [26], Aronsson and Johansson-Stenman 2008 [1]. 
equilibrium $^{5}$ ), few papers have investigated the implications of consumption externalities for welfare economics. ${ }^{6}$ The relative lack of interest from economists working on normative topics is usually motivated by a belief that only self-centered preferences should matter for social decisions (Goodin 1986 [24]). While it would indeed be very inappropriate to account for cruel or intrusive preferences, we believe that milder and more frequent forms of externalities should not necessarily be discarded (Fleurbaey 2012 [14]). Accounting for such externalities could lead to substantive welfare gains (Frank 2005 [22], Heath 2006 [25]), and it is therefore essential to understand how they should be incorporated into a measure of social welfare. We adress the question here from the perspective of fair social choice. ${ }^{7}$ The issue of deciding which type of preferences should be acknowledged, i.e. how to define the domain of admissible preferences, remains a difficult and thorny question. The answear may depend on the type of externality (positive vs negative, instrumental vs intrinsic, psychological vs technological) and the object of interest (is the externality directed towards poorer or richer individuals?). The results in this paper apply to various domains of preferences, and can therefore accomodate different opinions regarding this controversial issue.

The fair social choice approach usually relies on constructive proofs and the identification of intermediary allocations for which relevant principles can be applied. Here, the main difficulty comes from the impossibility of considering such intermediary allocations without controlling for everyone's welfare. We mitigate this issue by focusing on two simple specifications. In the first model, individuals care only about their own consumption and the average consumption in the economy (Average Externalities). The domain allows both for positive and negative externalities but may also be restricted so as to exclude either form of preferences (envious or altruistic). In the second model, individuals care only about their own consumption and their relative position in the consumption distribution (Positional Externalities). The externality, here, is always negative: relative position reflects social status and is valued positively.

For any allocation $x$, we define the egalitarian equivalent $u_{i}(x)$ as the quantity which leaves the individual indifferent between allocation $x$ and the egalitarian allocation where everyone receives $u_{i}(x)$. The concept of egalitarian equivalent was originally introduced by Pazner and Schmeidler (1978 [34]) in the model of fair allocation of a multi-dimensional good. In that setting, continuity is enough to ensure both existence and uniqueness because preferences are increasing in all dimensions. This is not the case in our model, as preferences may be decreasing with respect to others' consumption. However, the egalitarian equivalent is always well defined if we assume that preferences exhibit mild forms of negative externalities. In both models of Average and Positional

\footnotetext{
${ }^{5}$ Nogushi and Zame 2006 [33], Dufwenberger et al. 2011 [9].

${ }^{6}$ The existing literature focuses almost exclusively on allocation rules. Villar (1988 [46]), Nieto (1991 [32]) and Kolm (1995 [27]) look at the existence of Pareto optimal allocations in the division of a multi-dimensional good, while Velez (2014 [45]) shows the existence of envy-free allocations in the assignment of indivisible goods with monetary transfers. Only Decerf and Van der Linden ([8] 2014) follow the same approach as this paper, but their more general framework (multi-dimensional goods and a more general domain of preferences) does not allow for characterization results.

${ }^{7}$ For a comprehensive presentation of fair social choice, see Fleurbaey and Maniquet 2011 [17]. The approach has been applied to many different problems, including the fair allocation of multi-dimensional goods (Fleurbaey 2005 [12], 2007 [13], Fleurbaey and Maniquet 2008 [15]), public goods (Maniquet and Sprumont 2004 [29], 2005 [30]), indivisible goods (Maniquet 2008 [28]), the compensation of non transferable characteristics (Valletta 2009 [42]), the comparison of intergenerational allocations (Piacquadio 2014 [36]), the evaluation of income tax schedules (Fleurbaey and Maniquet 2006 [16]) and the comparison of allocations for populations of different sizes and preferences (Fleurbaey and Tadenuma $2014[19])$.
} 
externalities, we characterize the class of social ordering functions which give full priority to the individual with the lowest egalitarian equivalent in the economy. The characterization relies on four independent axioms, reflecting principles of efficiency, fairness and consistency.

The first requirement is Strong Pareto. Any allocation which weakly improves the welfare of all individuals should be considered a weak social improvement. If, in addition, the welfare of at least one individual is strictly improved, then the social comparison should also be strict. The Pareto principle applies either when all individuals benefit or when they all worsen from a given allocation. In order to construct a complete ordering, we also need to identify situations in which improving the welfare of some individual may be socially beneficial even when it is at the expense of some other individual. Defining such a fairness principle is not straightforward in our setting because the ordinal framework prevents inter-personal comparisons of utility. A classical strategy (Moulin 1992 [31]) consists in defining an individual benchmark level of welfare below (above) which agents may be considered disadvantaged (advantaged). Here, since individuals have no legitimate claims, a natural benchmark is to consider the situation where all consumption has been equally redistributed. The corresponding fairness requirement, Transfer to Disadvantaged, takes the form of a Pigou-Dalton transfer principle (Pigou 1912 [37]): any balanced transfer from some advantaged to some disadvantaged individual, while leaving the former advantaged and the latter disadvantaged, should be considered a social improvement. The third principle, Unchanged Contour Independence, requires social preferences to be independent from changes in individual preferences when indifference curves are left unmodified. The property is central in the literature because it allows to escape the Arrovian impossibility by weakening the axiom of Independence of Irrelevant Alternatives (Fleurbaey and Maniquet 2011 [17])). The social comparison between two allocations should not only depend on how all individuals compare these two allocations (IIA), but also on how all individuals compare these two allocations to all the other allocations (i.e. indifference curves). In the classical setting, imposing Strong Pareto, Unchanged-Contour Independence and some fairness requirements is enough to characterize the class of egalitarian equivalent maximin social ordering functions (Fleurbaey 2007 [13], Fleurbaey and Maniquet 2011, [17]). In contrast, social preferences may satisfy the three principles on our domains of preferences while completely ignoring both individual preferences and distributional considerations. Here, an additional Separability principle is needed to obtain the desired characterization. When there are no externalities, Separability requires the social comparison between two allocations to be unaffected if an individual who receives the same consumption bundle in the two allocations is given another identical bundle instead (Sen 1970 [38], Fleurbaey and Maniquet 2011 [17]). This requirement is unacceptable in our model as the other individuals may be affected by the change in the consumption of the indifferent individual. We introduce a new Separability requirement which allows the consumption of these other individuals to vary so as to remain indifferent to the original allocations.

Combining the mild Transfer to Disadvantaged with Strong Pareto, Unchanged-Contour Independence and Separability, forces social preferences to satisfy a much stronger egalitarian requirement. Any allocation which improves the welfare of some disadvantaged individual at the expense of some advantaged individual, while ensuring that (i) the latter remains advantaged and the former 
disadvantaged and (ii) all other individuals remain indifferent, should be considered a social improvement. The gain to the disadvantaged individual may now be infinitely smaller compared to the loss of the advantaged individual: social preferences exhibit infinite aversion to inequality.

On the domain of Average Externalities, this intermediary property leads to the main characterization theorem: any social ordering function which satisfies Strong Pareto, Unchanged Contour Independence, Separability and Transfer to Disadvantaged satisfies the maximin property with respect to egalitarian equivalents. If the lowest egalitarian equivalent at allocation $x$ is strictly larger than the lowest egalitarian equivalent at allocation $y$, then $x$ is stricty socially preferred to $y$. The result does not specify how society should compare allocations for which the lowest egalitarian equivalent is exactly the same. Different refinements may then be considered to obtain a complete ordering of all allocations, such as the corresponding maximin or leximin extensions. On the domain of Positional Externalities, these four principles are not enough to single out the class of maximin egalitarian equivalent social ordering functions. A stronger form of separability (allowing for variable population) is then required to obtain a characterization. The proofs of the two theorems rely on the particular geometry of the consumption space and do not generalize easily to more general forms of externalities. However, even in the general case, our social preferences still satisfy all the requirements on a broad domain of preferences, including well known models of other-regarding preferences such as Fehr and Schmidt 1999 [11] or Charness and Rabin 2002 [4].

The paper is organized as follows. In Section 2 we consider two simple examples to illustrate the general approach. In Section 3 we introduce the general framework, define the domain of admissible preferences and describe the main axioms. In Section 4 we study the domain of Average Externalities. In Section 5 we study the domain of Positional Externalities. Section 6 concludes. All proofs are gathered in Section 7.

\section{Illustrating the Approach}

\subsection{Sharing a Bequest}

A father wants to share a bequest between his two children, Ann and Bob. The bequests consists of two houses: a big one, of monetary value $v^{+}$, and a smaller one, of monetary value ${ }^{8} v^{-}<v^{+}$. Ideally, the father would like to give an equal share of the total amount $\Omega=v^{+}+v^{-}$to each of his children. Unfortunately, he does not have any money to compensate the child to which he would give the smaller house. The father could also choose to sell the houses, but it would be at a loss. The revenue $R$ he would get from their sale is strictly inferior to their real value: $R<\Omega$. Therefore, the father could either give the big house to one of his two children (and the small house to the other one) or sell the houses and share the revenue equally between them. We denote by $x^{1}, x^{2}$ and $x^{3}$ the corresponding allocations:

$$
x^{1}=\left(v^{-}, v^{+}\right), \quad x^{2}=\left(v^{+}, v^{-}\right) \quad \text { and } \quad x^{3}=\left(\frac{R}{2}, \frac{R}{2}\right) .
$$

Ann cares about the degree of inequality between herself and her brother. She would be willing to give up some of her own income for a decrease in the level of inequality. Bob, on the other hand, is only concerned by his own income. Their preferences are represented by the following utility

\footnotetext{
${ }^{8}$ The two children agree about the respective monetary value of the two houses.
} 
functions:

$$
v_{A}\left(x_{A}, x_{B}\right)=\sqrt{x_{A}}-\frac{1}{2}\left|x_{B}-x_{A}\right| \quad \text { and } \quad v_{B}\left(x_{A}, x_{B}\right)=x_{B} .
$$

Given his children's preferences, which of the three alternatives should the father choose?

For any allocation $x=\left(x_{A}, x_{B}\right)$, and any individual $i \in\{A, B\}$, we define individual $i$ 's egalitarian equivalent $u_{i}(x)$ as the quantity which would leave $i$ indifferent between allocation $x$ and the egalitarian allocation where both individuals receive $u_{i}(x)$. For example, at allocation $x^{1}=\left(v^{-}, v^{+}\right)$, Ann's egalitarian equivalent $u_{A}\left(x^{1}\right)$ must be such that:

$$
\sqrt{x_{A}^{1}}-\frac{1}{2}\left|x_{B}^{1}-x_{A}^{1}\right|=\sqrt{u_{A}\left(x^{1}\right)}+0 \quad \text { which implies } \quad u_{A}\left(x^{1}\right)=\left(\sqrt{v^{-}}-\frac{1}{2}\left(v^{+}-v^{-}\right)\right)^{2} .
$$

Similarly, Bob's egalitarian equivalent at $x^{1}$ must be such that $x_{B}^{1}=u_{B}\left(x^{1}\right)$, which simply yields $u_{B}\left(x^{1}\right)=v^{+}$. Note that the only information required for the computation of these numbers is the underlying ordinal preference relation. ${ }^{9}$ The egalitarian equivalent provides one particular way of measuring individual welfare from such ordinal preference relations.

We are interested in the class of social preferences which always give priority to the worst-off individual with respect to the egalitarian equivalent. Here, at allocation $x^{1}$, Ann is the worst-off individual in that respect, since $u_{A}\left(x^{1}\right)<u_{B}\left(x^{1}\right)$. At allocation $x^{2}$, Bob is now the worst-off individual, but his egalitarian equivalent is bigger than Ann's egalitarian equivalent at allocation $x^{1}$ :

$$
\min _{N} u_{i}\left(x^{2}\right)=u_{B}\left(x^{2}\right)=v^{-}>\left(\sqrt{v^{-}}-\frac{1}{2}\left(v^{+}-v^{-}\right)\right)^{2}=u_{A}\left(x^{1}\right)=\min _{N} u_{i}\left(x^{1}\right) .
$$

Our social preferences would thus recommend allocation $x^{2}$ over allocation $x^{1}$. At allocation $x^{3}$, both individuals are equally worse off and the egalitarian equivalent is simply equal to $R / 2$. Therefore, depending on whether the revenue $R$ is larger or smaller than $2 v^{-}$, our social preferences would either recommend $x^{3}$ or $x^{2}$ as the overall choice. Note that if the equal split allocation $(\Omega / 2, \Omega / 2)$ was available, it would always be chosen as the best allocation for any profile of preferences. However, because the bequest cannot be cut in half, other non-ideal allocations have to be considered. Our social preferences give one particular way of comparing such allocations.

\subsection{Conspicuous Consumption}

Two neighbors $i \in\{1,2\}$ consider buying a swimming pool. Neither of them really enjoys swimming, but both value the swimming pool as a way to display higher economic wealth. In this example, the swimming pool is a purely conspicuous good: individuals only derive utility from its consumption if the other neighbor doesn't own a swimming pool as well. Let $s_{i}$ denote the amount individual $i$ spends on such conspicuous consumption. Assume the utility attached to a vector $s=\left(s_{1}, s_{2}\right)$ is given by:

$$
\begin{aligned}
v_{i}\left(s_{i}, s_{j}\right) & =-s_{i}+\beta_{i} \quad \text { if } \quad s_{i}>s_{j} \quad \text { (i spends the most), } \\
& =-s_{i} \quad \text { if } \quad s_{i}=s_{j} \quad \text { (both spend the same), } \\
& =-s_{i}-\beta_{i} \quad \text { if } \quad s_{i}<s_{j} \quad \text { (i spends the least). }
\end{aligned}
$$

\footnotetext{
${ }^{9}$ The same values would be obtained for any other utility functions representing the same preference relations.
} 
where $\beta_{2}>\beta_{1}$. The parameter $\beta_{i}$ reflects individual i's taste for conspicuous consumption: the higher $\beta_{i}$, the more individual i cares about spending more than his neighbor. Assume there is only one type of swimming pool, of fixed price $p$ and let $\tau \geq 0$ be the constant tax rate on swimming pools. Tax revenue is redistributed equally between the two individuals. We consider the simultaneous game where individuals choose whether to buy a swimming pool or not.

$$
\text { Abstain Buy }
$$

$$
\begin{array}{ccc}
\text { Abstain } & (0,0) & \left(-\beta_{1}+p \frac{\tau}{2},-p\left(1+\frac{\tau}{2}\right)+\beta_{2}\right) \\
\text { Buy } & \left(-p\left(1+\frac{\tau}{2}\right)+\beta_{1},-\beta_{2}+p \frac{\tau}{2}\right) & (-p,-p)
\end{array}
$$

At equilibrium, when the tax rate is very low, $\tau<\beta_{1} / p-2$, both individuals buy a swimming pool. This outcome is clearly undesirable, as they would both get a higher payoff if neither of them decided to buy. This is exactly what happens if the tax rate is high enough, $\tau>\beta_{2} / p-2$. If the tax rate is intermediate, $\beta_{1} / p-2<\tau<\beta_{2} / p-2$, only the individual with the highest preference for status (individual 2) buys a swimming pool. How should society compare these different equilibria and choose the optimal tax rate?

Let $x_{i}$ denote individual $i$ 's post tax income. In the same fashion as before, we compute the egalitarian equivalent $u_{i}(x)$ as the quantity which leaves individual $i$ indifferent between allocation $x$ and the egalitarian allocation $\left(u_{i}(x), u_{i}(x)\right)$. Social welfare is aggregated by taking the lowest egalitarian equivalent in the economy, $W=\min u_{i}(x)$. As noted before, a very high tax rate $(W=0)$ is always preferable to a very low tax rate $(W=-p)$. However, the very high tax rate is only optimal if preferences are not too heterogeneous. Indeed, if individual 2 cares much more about status than individual $1, \beta_{2}-\beta_{1}>2 p$, then the intermediate tax rate $\tau^{*}=\left(\beta_{1}+\beta_{2}\right) / 2 p-1$ becomes optimal. ${ }^{10}$ In that case, individual 2 cares so much about consuming more than his neighbor that it becomes socially beneficial to induce the equilibrium where he buys a swimming pool (and individual 1 receives half of the tax revenue). Note that ignoring such conspicous preferences would lead to the Pareto dominated outcome (both in terms of resources and welfare) where both individuals buy the swimming pool.

The next section introduces the general setup and definitions.

\section{General Setup}

\subsection{Framework}

Let $\mathcal{N}$ be the infinite set of agents. A population is a finite set $N=\{1, \ldots, n\} \subset \mathcal{N}$ of agents. An allocation for population $N$ is a vector $x=\left(x_{1}, \ldots, x_{n}\right)$ in $\mathbb{R}^{n}$. For any such allocation, $\bar{x}=\sum_{i \in N} x_{i} / n$ denotes the average consumption ${ }^{11}$ in the economy. Each individual $i \in \mathcal{N}$ is

\footnotetext{
${ }^{10}$ Social Welfare is equal to $-p$ when the tax rate is very low, 0 when the tax rate is very high, and the minimum of $\frac{\tau}{2} p-\frac{\beta_{1}}{2}$ and $\left(1+\frac{\tau}{2}\right) p+\frac{\beta_{2}}{2}$ when the tax rate is intermediate. In this last case, social welfare is the highest for:

$$
\tau^{*}=\underset{\frac{\beta_{1}}{p}-2<\tau<\frac{\beta_{2}}{p}-2}{\arg \max } \min \left(\frac{\tau}{2} p-\frac{\beta_{1}}{2},-\left(1+\frac{\tau}{2}\right) p+\frac{\beta_{2}}{2}\right)=\frac{\beta_{1}+\beta_{2}}{2 p}-1 .
$$
}

and the corresponding social welfare is equal to $W^{*}=\left(\beta_{2}-\beta_{1}\right) / 4-p / 2$.

${ }^{11}$ Throughout the paper we use the term consumption to refer to the amount allocated to each individual. 
characterized by a collection of ordinal preference relations $\left\{R_{i}^{n}\right\}_{n>0}$, where $R_{i}^{n}$ gives i's preferences over the set of allocations $\mathbb{R}^{n}$ (corresponding to populations of size ${ }^{12} n$ ). Preferences are strictly increasing in personal consumption but may be either increasing or decreasing with respect to others' consumption. For any preference relation $R_{i}^{n}$, the corresponding strict preference and indifference relations are denoted by $P_{i}^{n}$ and $I_{i}^{n}$ respectively. A preference profile for population $N$ is a list of individual preference relations over $\mathbb{R}^{n}$ for individuals in $N, R_{N}=\left(R_{1}^{n}, \ldots, R_{n}^{n}\right)$.

An economy $E$ is defined by a population $N$ and a profile of preferences $R_{N}, E=\left(N, R_{N}\right)$. A social ordering is a complete and transitive binary relation over the set of allocations $\mathbb{R}^{n}$. A social ordering function $R\left(\right.$.) associates every economy $E=\left(N, R_{N}\right)$ with a social ordering $R(E)$ over $\mathbb{R}^{n}$. Our objective is to construct and characterize social ordering functions which satisfy interesting ethical properties.

When the population is fixed (sections 3 and 4 ), we abuse notation by simply writing $R_{i}$ instead of $R_{i}^{n}$. We allow population to vary in Section 5 (Positional Externalities). ${ }^{13}$

\subsection{Domain of Preferences}

To what extent should society respect envious preferences in evaluating social welfare? In their analysis of general equilibrium, Dufwenberg et al. (2011 [9]) suggest the following restriction: ${ }^{14}$

Definition 1. A preference profile $R_{N}$ satisfies Social Monotonicity if for any allocation $x$ in $\mathbb{R}^{n}$ and any $\delta>0$ there exists $z$ in $\mathbb{R}^{n}$ such that:

$$
\sum_{i \in N} z_{i}=\delta \quad \text { and } \quad x+z P_{i} x \quad \forall i \in N .
$$

A preference profile satisfies Social Monotonicity if it is always possible to distribute additional resources so as to make everyone strictly better off. Social Monotonicity provides a natural restriction on the extent of negative externalities. In particular, it ensures that any Pareto efficient allocation is always achievable as a Walrasian equilibrium (Second Welfare Theorem in [9]). However, because it applies to profiles of preferences as a whole, it still allows for extremely envious preferences if there exist other altruistic individuals in the economy. Imagine the case of a two-person economy where individual 1 is extremely envious while individual 2 is just slightly altruistic.

$$
\left.u_{1}(x)=\varepsilon x_{1}-x_{2} \quad \text { and } \quad u_{2}(x)=x_{2}+\varepsilon x_{1} \quad \text { for some small } \varepsilon \in\right] 0,1[
$$

In that economy, giving all additional consumption $\delta$ to individual 1 would make both individuals 1 and 2 strictly better off. Social Monotonicity is satisfied despite individual 1's extremely envious preferences.

In order to avoid such an extreme form of negative externalities, we choose to impose restrictions directly at the level of individual preference relations (as opposed to preference profiles).

Definition 2. A preference relation $R_{i}$ satisfies Reasonable Envy if:

(I) For any allocation $x$ in $\mathbb{R}^{n}$ and any $\delta>0: \quad x+(\delta, \ldots, \delta) P_{i} x$.

\footnotetext{
${ }^{12}$ We presuppose anonymity of preferences, which means individual preferences are the same for populations of same sizes.

${ }^{13}$ Since preferences change as $n$ varies, some additional conditions will then be imposed to ensure consistency of preferences.

${ }^{14}$ Their restriction is formulated in a multi-dimensional framework.
} 
(II) There exists $-\infty<m<M<+\infty$ such that for any allocation $x$ in $\mathbb{R}^{n}$ and any $\delta>0$ :

$$
x_{i}<m \text { or } x_{i}>M \Rightarrow x P_{i} x-\delta e_{j} \quad \forall j \neq i .
$$

Condition (I) means one should always be strictly better off when the consumption of all individuals increases by the same quantity. Condition (II) forbids negative externalities for arbitrarily extreme (either low or high) levels of consumptions. It should always be possible to find a large (small) enough quantity $M<+\infty(m>-\infty)$ above (below) which individuals do not envy others' consumption anymore. Both conditions are always satisfied when the externality is positive but may be violated for strong forms of negative interdependencies. A preference profile $R_{N}$ belongs to $\mathcal{R}^{R E}$ if all preference relations in $R_{N}$ are continuous and satisfy Reasonable Envy.

\subsection{Egalitarian Equivalent}

We define individual i's egalitarian equivalent at allocation $x \in \mathbb{R}^{n}$ as the quantity $u_{i}(x)$ which leaves $i$ indifferent between allocation $x$ and the egalitarian allocation where everyone receives $u_{i}(x)$ :

$$
x I_{i}\left(u_{i}(x), \ldots, u_{i}(x)\right) .
$$

The concept of egalitarian equivalent was originally introduced in the model of fair division of a multi-dimensional good (Pazner and Schmeidler 1978 [34]). In that setting, continuity is enough to ensure both existence and uniqueness of the egalitarian equivalent because preferences are increasing in all dimensions. This is not the case in our model, as preferences may be decreasing with respect to others' consumption. ${ }^{15}$ However, the egalitarian equivalent is always well defined if preferences exhibit the mild forms of externalities characterized by Reasonable Envy.

Proposition 1. On $\mathcal{R}^{R E}$, the egalitarian equivalent is well defined.

The egalitarian equivalent provides a numerical representation of individual preferences: $x R_{i} y \Leftrightarrow$ $u_{i}(x) \geq u_{i}(y)$. We are interested in the class of social ordering functions which give absolute priority to the individual with the lowest egalitarian equivalent in the economy.

Definition 3. A social ordering function $R($.$) is an egalitarian equivalent maximin social ordering$ function if for any $x, y \in \mathbb{R}^{n}$ :

$$
\min _{N} u_{i}(x)>\min _{N} u_{i}(y) \quad \Rightarrow \quad x P(E) y
$$

The definition does not specify what should happen in case of equality. Different refinements may then be considered to obtain a complete ordering of all allocations. Notable refinements include the maximin, where such equality implies social indifference $(x I(E) y)$, and the leximin, where the equality is broken by looking at the second worst-off individuals, then third worst-off etc...

\subsection{Axioms}

3.4.1. Efficiency. We impose the strong version of the Pareto principle.

\footnotetext{
${ }^{15}$ The egalitarian equivalent is always well defined for positive externalities. Existence and uniqueness are still satisfied for mild form of negative externalities, such as in Fehr and Schmidt's model of inequality aversion ([11] 1999) but may be violated for stronger forms of envy. Consider for example $x R_{i} y$ iff $\frac{x_{i}}{\bar{x}} \geq \frac{y_{i}}{\bar{y}}$. Whenever $x_{i}>\bar{x}$, the egalitarian equivalent does not exist.
} 


\section{Axiom 1. Strong Pareto}

For any economy $E=\left(N, R_{N}\right)$ and any allocations $x$ and $y$ in $\mathbb{R}^{n}$, if $y R_{i} x$ for all $i$ in $N$, then $y R(E) x$. If, in addition, $y P_{j} x$ for some $j$ in $N$, then $y P(E) x$.

If all individuals prefer allocation $y$ to allocation $x$, then it should also be the case from a social point of view. If, in addition, at least one of them prefers $y$ strictly, then the social comparison should also be strict.

3.4.2. Fairness. The Pareto principle applies either when all individuals benefit or when they all worsen from a given allocation. In order to construct a complete ordering, we also need to identify situations in which improving the welfare of some individual may be socially beneficial even when it is at the expense of some other individual. Defining such a fairness principle is not straightforward in our setting because the ordinal framework prevents inter-personal comparisons of utility. A classical strategy (Moulin 1992 [31]) consists of defining an individual benchmark level of welfare below (above) which agents may be considered disadvantaged (advantaged). Here, since individuals have no legitimate claims, a natural benchmark is to consider the situation where all consumption has been equally redistributed.

Definition 4. For any allocation $x$ in $\mathbb{R}^{n}$, we define the set of disadvantaged individuals by:

$$
D\left(x, R_{N}\right)=\left\{i \in N \mid(\bar{x}, \ldots, \bar{x}) P_{i} x\right\} .
$$

Someone is disadvantaged if he strictly prefers the equal split allocation $(\bar{x}, \ldots, \bar{x})$ to the current allocation $x$. We say that someone is advantaged when he is not disadvantaged, $A\left(x, R_{N}\right)=$ $N \backslash D\left(x, R_{N}\right)$. The corresponding fairness requirement takes the form of a Pigou-Dalton transfer principle.

\section{Axiom 2. Transfer to Disadvantaged}

For any economy $E=\left(N, R_{N}\right)$, any allocations $x$ and $y$ in $\mathbb{R}^{n}$, any individuals $i \in D\left(x, R_{N}\right) \cap$ $D\left(y, R_{N}\right), j \in A\left(x, R_{N}\right) \cap A\left(y, R_{N}\right)$ and any $\Delta>0$ :

$$
\left[y_{i}=x_{i}+\Delta, \quad y_{j}=x_{j}-\Delta, \quad y_{k}=x_{k} \forall k \neq i, j, \quad y R_{k} x \quad \forall k \neq i\right] \Rightarrow \quad y R(E) x .
$$

Any balanced transfer from some advantaged individual $j$ to some disadvantaged individual $i$, while ensuring that (i) $j$ remains advantaged and $i$ remains disadvantaged, and (ii) no individual besides $j$ is made worse off, should be considered a social improvement. ${ }^{16}$

3.4.3. Independence. The third principle requires social comparison to be independent from changes in individual preferences when indifference curves are left unmodified. The property, a weakening of the classical axiom of independence of irrelevant alternatives, is central in the literature of fair social choice (Fleurbaey and Maniquet 2008 [15], 2011 [17]).

\section{Axiom 3. Unchanged-Contour Independence}

\footnotetext{
${ }^{16}$ Note that on both domains of Average and Positional Externalities, this last requirement is superfluous because such a balanced transfer necessarily leaves all individuals but $j$ better off.
} 
For any economies $E=\left(N, R_{N}\right)$ and $E^{\prime}=\left(N, R_{N}^{\prime}\right)$ and any allocations $x, y$ in $\mathbb{R}^{n}$ :

$$
\left[\mathbf{I}\left(x, R_{i}^{\prime}\right)=\mathbf{I}\left(x, R_{i}\right) \text { and } \mathbf{I}\left(y, R_{i}^{\prime}\right)=\mathbf{I}\left(y, R_{i}\right) \forall i \in N\right] \Rightarrow\left[y R(E) x \Leftrightarrow y R\left(E^{\prime}\right) x\right] .
$$

If profiles $R_{N}$ and $R_{N}^{\prime}$ are such that indifference curves coincide at allocations $x$ and $y$ for all individuals, then the social comparison between $x$ and $y$ should be the same for economies $\left(N, R_{N}\right)$ and $\left(N, R_{N}^{\prime}\right)$. The social comparison between $x$ and $y$ should not only depend on how all individuals compare $x$ and $y$, as advocated by Independence of Irrelevant Alternatives, but also on how all individuals compare $x$ and $y$ to any of the other allocations. This corresponds exactly to the information contained in the indifference curves.

Our second independence requirement takes the form of a Separability principle. In the classical setting, the usual axiom requires the social comparison between two allocations to be unaffected if an individual who receives the same consumption bundle in two allocations is given another identical bundle instead ${ }^{17}$ (Fleurbaey and Maniquet 2011 [17]). This requirement is unacceptable in our model, as the other individuals may be affected by the change in the consumption of the indifferent individual. We introduce a new Separability requirement where the consumption of these other individuals is allowed to vary so as to remain indifferent to their consumption bundles in the original allocations.

\section{Axiom 4. Separability}

For any economy $E=\left(N, R_{N}\right)$, any $x, y, x^{\prime}, y^{\prime}$ in $\mathbb{R}^{n}$ and any $k \in N$ :

$$
\left[x I_{k} y, \quad x^{\prime} I_{k} y^{\prime}, \quad x I_{i} x^{\prime} \text { and } y I_{i} y^{\prime} \forall i \in N \backslash\{k\}\right] \Rightarrow\left[x R(E) y \quad \Leftrightarrow \quad x^{\prime} R(E) y^{\prime}\right] .
$$

If individual $k$ is indifferent between allocations $x$ and $y$, and there exist two allocations $x^{\prime}$ and $y^{\prime}$ such that (i) $k$ is also indifferent between $x^{\prime}$ and $y^{\prime}$ and (ii) all other individuals in $N$ are indifferent both between allocations $x$ and $x^{\prime}$ and between allocations $y$ and $y^{\prime}$, then the social comparison between $x^{\prime}$ and $y^{\prime}$ should be the same as the social comparison between $x$ and $y$. This axiom extends the classical separability requirement by taking into account indifferences. The requirement is illustrated for the domain of Average Externalities in the next section (Figure 2).

Assuming Continuity and Reasonable envy, the egalitarian equivalent leximin social ordering function satisfies all requirements.

Proposition 2. On $\mathcal{R}^{R E}$, the egalitarian equivalent leximin social ordering function satisfies Strong Pareto, Transfer to Disadvantaged, Unchanged-Contour Independence and Separability.

On $\mathcal{R}^{R E}$, any egalitarian equivalent maximin social ordering function satisfies Weak Pareto, Transfer to Disadvantaged and Unchanged-Contour Independence, but may violate Strong Pareto and Separability.

We turn to the model of Average Externalities.

${ }^{17}$ In classical social choice, this requirement is generally referred to as Independence from Indifferent Individuals (Sen $1970,[38])$. 


\section{Average Externalities}

\subsection{Framework}

Individuals care only about their own consumption $x_{i}$ and the average consumption ${ }^{18}$ in the economy $\bar{x}=\sum_{i \in N} x_{i} / n$.

Definition 5. A preference relation $R_{i}$ exhibits Average Externalities if there exists a continuous preference relation $\hat{R}_{i}$ over $\mathbb{R}^{2}$ such that:

$$
x R_{i} y \quad \Leftrightarrow \quad\left(x_{i}, \bar{x}\right) \hat{R}_{i}\left(y_{i}, \bar{y}\right) .
$$

In what follows, we abuse notation by using $R_{i}$ instead of $\hat{R}_{i}$ when comparing consumption bundles $\left(x_{i}, \bar{x}\right)$. For technical purposes, we also need to make the additional assumption that individuals are not infinitely altruistic.

Definition 6. A preference relation $R_{i}$ satisfies Finite Altruism if:

$$
\inf _{x \in \mathbb{R}^{n}, \delta>0}\left\{\varepsilon \geq 0 \mid x-\delta e_{i}+\varepsilon \delta e_{-i} P_{i} x\right\}>0 .
$$

Finite altruism means that the marginal rate of substitution cannot become arbitrarily small.

Definition 7. A preference profile $R_{N}$ is in the domain of Average Externalities $\mathcal{R}^{A}$ if all preference relations in $R_{N}$ satisfy Reasonable Envy, Average Externalities and Finite Altruism.

The model allows both for envious and altruistic preferences, i.e. negative and positive externalities with respect to the average consumption $\bar{x} .{ }^{19}$ We do not assume convexity of preferences, but all the results are also valid on the subdomain of convex preferences. ${ }^{20}$

\subsection{Consumption Space}

Graphically, a consumption bundle is represented by a point in the plane where the first (horizontal) coordinate gives the individual's personal consumption and the second (vertical) coordinate gives the average consumption in the economy. Here, the quantities consumed by all individuals are not independent, so the consumption bundles corresponding to a given allocation may not be chosen freely in the consumption space. These bundles must always be located on the same horizontal line (all individuals face the same average consumption) and their average horizontal coordinate must always be equal to their common vertical coordinate. The egalitarian equivalent is given by the intersection point of the indifference curve with the diagonal $x_{i}=\bar{x}$. Condition (I) in Reasonable Envy means that individuals are always strictly better off when their own consumption increases by more (or the same) than the average consumption. As a result, the slope of indifference curves is always either negative or strictly superior to 1. Finite Altruism implies that indifference curves are never asymptotically flat. The consumption space is illustrated in Figure 1.

\footnotetext{
${ }_{18}$ Alternatively, we could assume individuals care about the average consumption in the rest of the economy $\bar{x}_{-i}=$ $\sum_{k \neq i} x_{k} /(n-1)$. We retain the first specification because it is easier to manipulate but the two models are formally equivalent.

${ }^{19}$ The sign of the externality may depend on the region of the consumption space. Someone could for example be envious when his consumption level is below the average consumption, but altruistic when it is above.

${ }^{20}$ Note in particular that all the preferences we artificially construct in the proofs are convex.
} 


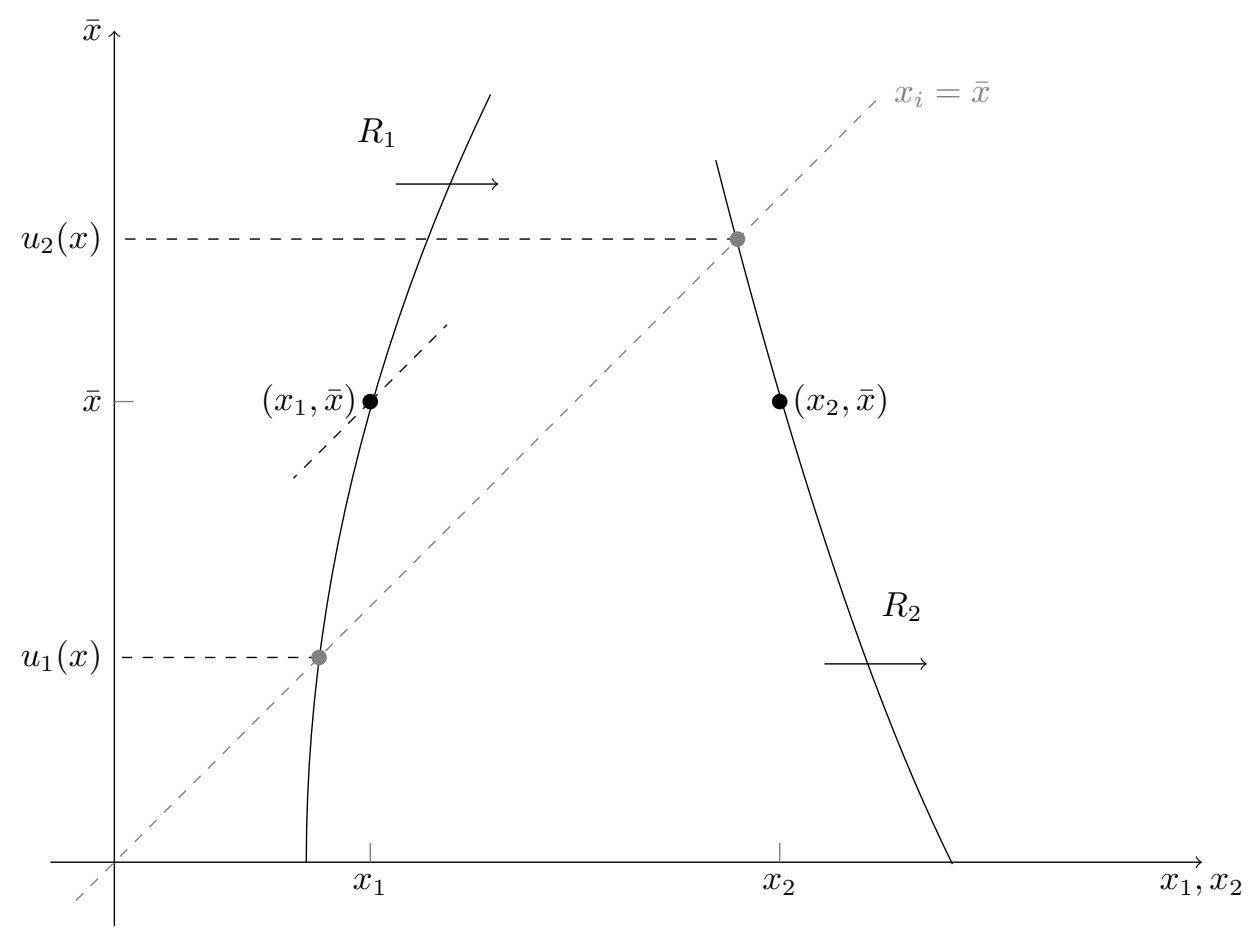

Figure 1. In this example individual 1's indifference curve is upward sloping, meaning 1 values the average consumption negatively (envious preferences), while individual 2's indifference curve is downward sloping, he values the average consumption positively (altruistic preferences).

A natural consequence of Reasonable Envy for the model of Average Externalities is that lower average allocations never Pareto dominate higher average allocations: there is no Pareto efficient way of destroying resources. ${ }^{21}$ Let's assume, by contradiction, that there exist two allocations $x$ and $y$ such that $\bar{x}<\bar{y}$ and $x$ Pareto dominates $y$. Then, since 1 must necessarily prefer $x$ to $y$, condition (I) in Reasonable Envy means that $y_{1}<x_{1}+(\bar{y}-\bar{x})$, which then implies $y_{2}>x_{2}+(\bar{y}-\bar{x})$. By Reasonable Envy again, 2 must necessarily prefer $y$ to $x$, a contradiction.

\subsection{Axioms}

On $\mathcal{R}^{A}$, individuals are disadvantaged when they receive strictly less than the average consumption: ${ }^{22} D\left(x, R_{N}\right)=\left\{i \in N \mid x_{i}<\bar{x}\right\}$. Accordingly, we ignore preferences and write $D(x)$ instead of $D\left(x, R_{N}\right)$. As noted earlier, because the transfer in Transfer to Disadvantaged is balanced, the average consumption does not vary so that all the other individuals in the economy remain unaffected by the transfer. ${ }^{23}$ The requirement that no individual besides $j$ is made worse off is therefore superfluous.

\footnotetext{
${ }^{21}$ Decerf and Van der Linden ([8] 2014) refer to this property as No Unanimous Destruction of Resources. For the same reason, two different allocations can never be Pareto indifferent on $\mathcal{R}^{A}$.

${ }^{22}$ Follows from the strict monotonicity of individual preferences with respect to $x_{i},(\bar{x}, \bar{x}) P_{i}\left(x_{i}, \bar{x}\right) \Leftrightarrow x_{i}<\bar{x}$. .

${ }^{23}$ The standard Pigou-Dalton transfer principle used in the theory of inequality measurement (Pigou 1912 [37]) requires any transfer from some richer to some poorer individual (while preserving their relative order) to be considered a social improvement. It reflects a stronger form of resource egalitarianism than Transfer to Disadvantaged because it also applies to pairs of individuals who are either both advantaged or both disadvantaged. In contrast with the classical setting, where Transfer is incompatible with Pareto (for multidimensional goods), the two requirements can be satisfied by the same SOF on $\mathcal{R}^{A}$. Consider $x R(E) y$ iff $\bar{x} \geq \bar{y}$.
} 
In the classical setting, imposing Strong Pareto, Unchanged-Contour Independence and some fairness requirements ${ }^{24}$ is enough to characterize the class of egalitarian equivalent maximin functions (Fleurbaey 2007 [13], Fleurbaey and Maniquet 2011, [17]). In contrast, social preferences may satisfy the three principles on our domains of preferences while completely ignoring both individual preferences and distributional considerations. ${ }^{25}$ Here, the additional Separability principle is required to obtain a characterization. The application of Separability on $\mathcal{R}^{A}$ is illustrated in Figure 2.

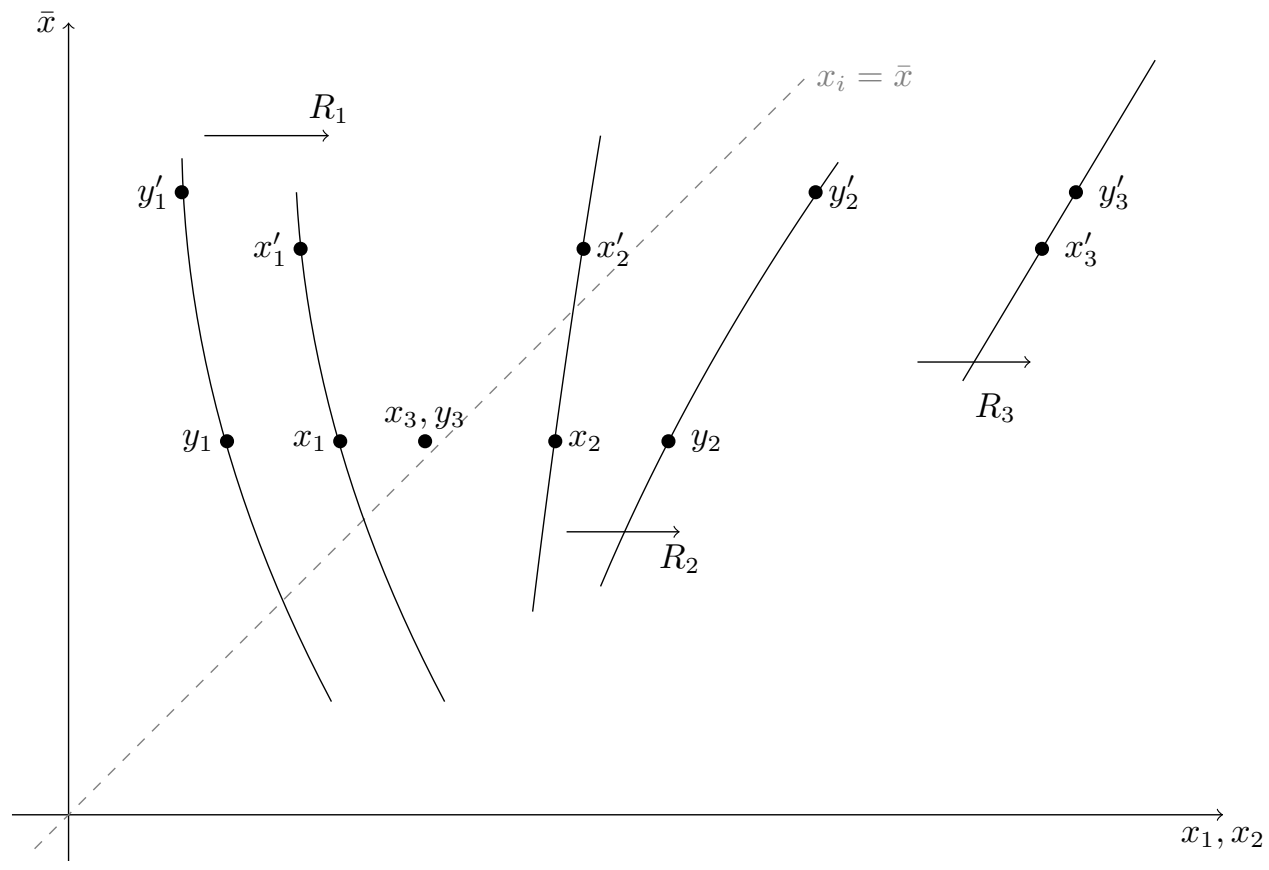

Figure 2. In this example, individual 3 is indifferent both between $x$ and $y$ and between $x^{\prime}$ and $y^{\prime}$, while individuals 1 and 2 are indifferent both between $x$ and $x^{\prime}$ and between $y$ and $y^{\prime}$. In that situation, Separability recommends the social comparison between $x$ and $y$ to be the same as between $x^{\prime}$ and $y^{\prime}$.

\subsection{From Transfer to Priority}

Our fairness principle, Transfer to Disadvantaged, only applies to allocations of equal size because it involves balanced transfers. A stronger property would be to require that any allocation which improves the welfare of some disadvantaged individual at the expense of some advantaged individual, while ensuring that (i) the latter remains advantaged and the former disadvantaged and (ii) all the other individuals remain indifferent, be considered a social improvement. The corresponding axiom writes.

\section{Axiom 5. Priority to Disadvantaged}

For any economy $E=\left(N, R_{N}\right)$, any allocations $x$ and $y$ in $\mathbb{R}^{n}$, and any individuals $i \in D(x) \cap D(y)$, $j \in A(x) \cap A(y):$

\footnotetext{
${ }^{24}$ In Fleurbaey 2007 [13] the fairness requirement takes the form of a mean support dominance property, while in Fleurbaey and Maniquet 2011 ([17]) a combination of two transfer principles.

${ }^{25}$ Consider again $x R(E) y$ iff $\bar{x} \geq \bar{y}$.
} 


$$
\left[y P_{i} x, \quad x P_{j} y \quad \text { and } \quad x I_{k} y \forall k \neq i, j\right] \Rightarrow y R(E) x .
$$

As the average consumption may now vary, we also need to ensure all the other individuals remain indifferent to the initial allocation. Priority to Disadvantaged is a much stronger requirement than Transfer to Disadvantaged because it involves an infinite aversion to inequality: the gain of the disadvantaged individual may now be infinitely small compared to the loss of the advantaged individual.

On the domain of Average Externalities, if a social ordering function satisfies Strong Pareto, Unchanged-Contour Independence and Separability, imposing Transfer to Disadvantaged forces us to satisfy Priority to Disadvantaged.

Proposition 3. On $\mathcal{R}^{A}$, for $n \geq 3$, if a social ordering function satisfies Strong Pareto, Unchanged Contour Independence, Separability and Transfer to Disadvantaged then it also satisfies Priority to Disadvantaged.

As alluded before, the implication from zero to infinite aversion to inequality is a classical result in fair social choice. However, in contrast with the usual setting, where Pareto, Transfer ${ }^{26}$ and Unchanged-Contour Independence are enough to generate full priority, we need to impose the additional Separability requirement. We can now state the characterization result.

\subsection{The Egalitarian Equivalent SOF}

Any social ordering function which satisfies Strong Pareto, Unchanged Contour Independence, Separability and Transfer to Disadvantaged must be an egalitarian equivalent maximin social ordering function.

Theorem 1. On $\mathcal{R}^{A}$, for $n \geq 3$, if a social ordering function $R($.$) satisfies Strong Pareto,$ Unchanged Contour Independence, Separability and Transfer to Disadvantaged, then for all $E=\left(N, R_{N}\right)$ and $x, y$ in $\mathbb{R}^{n}$ :

$$
\min _{i \in N} u_{i}(x)>\min _{i \in N} u_{i}(y) \Rightarrow x P(E) y .
$$

If the lowest egalitarian equivalent at allocation $x$ is strictly larger than the lowest egalitarian equivalent at allocation $y$, then $x$ is strictly socially preferred to $y$. The characterization result does not specify how society should compare allocations for which the lowest egalitarian equivalent is exactly the same. Different refinements may then be considered to obtain a complete ordering of all allocations. Conversely, any strict maximin egalitarian equivalent social ordering function satisfies Weak Pareto, Transfer to Disadvantaged and Unchanged-Contour Independence. However, Strong Pareto and Separability may not be satisfied, as in the case of the maximin social ordering function $^{27}$, which violates both. The leximin refinement satisfies all axioms, but a characterization cannot be obtained using only these four requirements. ${ }^{28}$ Using Priority to Disadvantaged (implied

\footnotetext{
${ }^{26}$ In the classical multi-dimensional setting, the closest axiom to Transfer to Disadvantaged would be Equal Split Transfer. Combining Strong Pareto, Unchange-Contour Independence and Equal-Split Transfer leads to Equal Split Priority (Fleurbaey and Maniquet 2011 [17]).

${ }^{27} \min _{N} u_{i}(x)=\min _{N} u_{i}(y)$ implies $x I(E) y$.

${ }^{28}$ Other social ordering functions also satisfy all the requirements. One such example, as suggested in Fleurbaey and Maniquet 2011 [17] (for a different context), consists in applying a different leximin social ordering to break possible ties in the original leximin social ordering function.
} 
by Proposition 2), the first step of the proof consists in showing that (i) society always respects the opinion of disadvantaged individuals when they all agree about the comparison between two allocations (Disadvantaged Unanmity) and (ii) any egalitarian allocation is strictly preferred to all other allocations of same size (Equal Split Selection). The Theorem then obtains by joint application of these two properties with Strong Pareto. The four requirements are logically independent.

\subsection{Alternative Domains}

The domain of preferences may be further restricted to allow only for positive or only for negative externalities, reflecting alternative views about how society should account for other-regarding preferences. We say that a preference profile $R_{N}$ satisfies Positive (Negative) Externalities if all preference relations in $R_{N}$ are increasing (decreasing) with respect to average income.

Definition 8. A preference profile $R_{N}$ is in the domain of Positive Average Externalities $\mathcal{R}^{P A}$ if all preference relations in $R_{N}$ satisfy Average Externalities, Positive Externalities and Finite Altruism.

A preference profile $R_{N}$ is in the domain of Negative Average Externalities $\mathcal{R}^{N A}$ if all preference relations in $R_{N}$ satisfy Average Externalities, Negative Externalities and Reasonable Envy.

Note that any profile in either $\mathcal{R}^{P A}$ or $\mathcal{R}^{N A}$ is also in $\mathcal{R}^{A}$. The characterization of strict maximin egalitarian equivalent Social Ordering Functions is valid on both subdomains of preferences.

Corrolary 1. Theorem 1 holds on $\mathcal{R}^{P A}$ and $\mathcal{R}^{N A}$.

We turn to the model of Positional Externalities.

\section{Positional Externalities}

\subsection{Framework}

For any population $N$, define individual $i$ 's relative position at allocation $x \in \mathbb{R}^{n}$ by:

$$
p_{i}^{N}(x)=\frac{1}{n-1}\left[\sharp\left\{j \in N \backslash\{i\} \mid x_{j}<x_{i}\right\}+\frac{1}{2} \sharp\left\{j \in N \backslash\{i\} \mid x_{i}=x_{j}\right\}\right] .
$$

The highest ranked individual is always given a relative position of 1 while the lowest ranked individual is always given a relative position of 0 (if alone at that position). When there is no possible confusion, we write $p_{i}(x)$ instead of $p_{i}^{N}(x)$.

We assume individuals only care about their own consumption $x_{i}$ and their relative position $p_{i}(x)$. We denote by $\left(x_{i}, p_{i}(x)\right)$ individual i's consumption bundle at allocation $x$.

Definition 9. Individual preferences $\left\{R_{i}^{n}\right\}_{n \in \mathbb{N}^{*}}$ exhibit Positional Externalities if there exists a continuous preference relation $\tilde{R}_{i}$ over $\mathbb{R}^{2}$ such that:

$$
x R_{i}^{n} y \quad \Leftrightarrow \quad\left(x_{i}, p_{i}^{N}(x)\right) \tilde{R}_{i}\left(y_{i}, p_{i}^{N}(y)\right) \quad \forall N \subset \mathcal{N} .
$$

where $\tilde{R}_{i}$ is strictly increasing in both $x_{i}$ and $p_{i}^{N}(x)$. 
In what follows, we abuse notation by using $R_{i}$ instead of $\tilde{R}_{i}$ when referring to consumption bundles. Note that preference relations are assumed to be strictly increasing with relative position. Individuals all want to have a higher status, as reflected by their relative position, but their willingness to trade absolute consumption for a higher position may vary.

Definition 10. A preference profile $R_{N}$ is in the Positional Domain $\mathcal{R}^{P}$ if all preference relations in $R_{N}$ satisfy Reasonable Envy ${ }^{29}$ and Positional Externalities.

A consumption bundle is represented by a point in $\mathbb{R} \times[0,1]$ where the first (horizontal) coordinate gives the individual consumption and the second (vertical) coordinate gives his relative position. All the consumption bundles corresponding to a given allocation must always be located on an upwardsloping path, as richer individuals necessarily have a higher relative position than poorer individuals. Indifference curves are downward sloping because preferences are increasing with respect to both personal consumption and relative position. Graphically, the egalitarian equivalent is given by the horizontal coordinate of the intersection point between the individual's indifference curve and the horizontal line $p_{i}=1 / 2$. The consumption space is illustrated in Figure 3 .

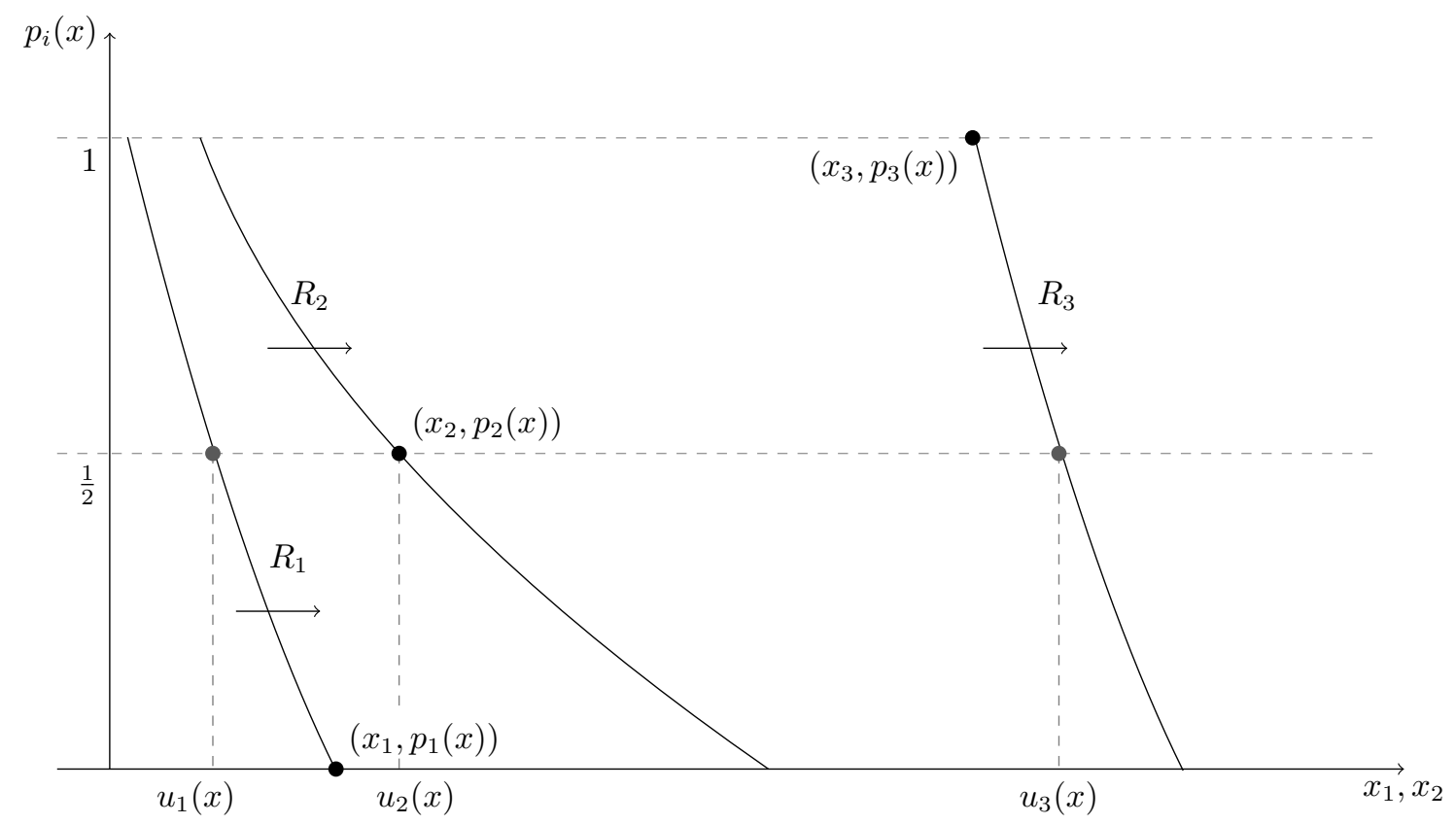

Figure 3. Consumption Space in the model of Positional Externalities. Indifference curves are downward sloping on $\mathcal{R}^{P}$ because individuals value positively both $x_{i}$ and $p_{i}(x)$.

Imposing Positional Externalities alone is enough to satisfy No unanimous Destruction of Resources: lower average allocations never Pareto dominate higher average allocations. By contradiction, assume all individuals prefer strictly $x$ to $y$ and $\bar{x}<\bar{y}$. Then necessarily, one individual must get a smaller consumption at $x$ than at $y$. But since that individual must also prefer $x$ to $y$, it must be that his relative position has increased. This can only happen if some richer individual (in

\footnotetext{
${ }^{29}$ Note that when a profile $R_{N}$ satisfies Positional Externalities, Social Monotonicity as well as Condition (I) in Reasonable Envy are trivially satisfied. For any allocation $x \in \mathbb{R}^{n}$ and $\delta>0$, just consider the allocation $z$ where all individuals are given an additional $\delta / n: z_{i}=x_{i}+\delta / n \forall i \in N$. Since both $p_{i}(x)=p_{i}(z)$ and $z_{i}>x_{i}$ for all $i \in N$, they must all prefer (strictly) $z$ to $x$ : Social Monotonicity is satisfied.
} 
allocation $y$ ) is now poorer than him. In turn, since that individual must prefer $x$ to $y$, it should also be the case that his own relative position has increased. Repeating this argument, we finally get to the conclusion that the relative position of the highest consumption individual in allocation $y$ must also have increased when going to allocation $x$. However, this is impossible since that individual is already at the top position. We thus get a contradiction and conclude that $\bar{x}>\bar{y}$ when $x$ Pareto dominates $y$.

\subsection{Axioms}

On the Positional domain, imposing the four previous axioms is not enough to single out the class of egalitarian equivalent maximin social ordering functions. ${ }^{30}$ We need to replace Separability by a stronger Separation principle, which is the reason why the model requires a variable population setting. The axiom is very similar to Separability, but instead of demanding the social comparison to be unaffected by changes in the welfare of indifferent individuals, we require the social comparison to be unaffected by the addition or deletion of such indifferent individuals from the economy.

Axiom 6. Separation: For any economy $E=\left(N, R_{N}\right)$, any $A, B \subset N$, any $x, y \in \mathbb{R}^{a}$, and any $x^{\prime}, y^{\prime} \in \mathbb{R}^{b}$ :

$$
\begin{aligned}
& {\left[x I_{k} y \forall k \in A \backslash B, \quad, x^{\prime} I_{k} y^{\prime} \forall k \in B \backslash A, \quad x I_{i} x^{\prime} \text { and } y I_{i} y^{\prime} \quad \forall i \in A \cap B\right]} \\
& \Rightarrow \quad\left[x R\left(A, R_{A}\right) y \Leftrightarrow x^{\prime} R\left(B, R_{B}\right) y^{\prime}\right] .
\end{aligned}
$$

For any two subsets $A, B \subset N$, any allocations $x$ and $y$ in $\mathbb{R}^{a}$ and any allocations $x^{\prime}$ and $y^{\prime}$ in $\mathbb{R}^{b}$ if (i) all individuals in $A \backslash B$ are indifferent between $x$ and $y$, (ii) all individuals in $B \backslash A$ are indifferent between $x^{\prime}$ and $y^{\prime}$ while (iii) all individuals in $A \cap B$ are indifferent both between $x$ and $x^{\prime}$ and between $y$ and $y^{\prime}$, then the social comparison between $x$ and $y$ in economy $\left(A, R_{A}\right)$ should be the same as the social comparison between $x^{\prime}$ and $y^{\prime}$ in economy $\left(B, R_{B}\right)$.

On $\mathcal{R}^{P}$ the subset of disadvantaged individuals is given by:

$$
D\left(x, R_{N}\right)=\left\{i \in N \mid(\bar{x}, \ldots, \bar{x}) P_{i} x\right\}=\left\{i \in N \mid\left(\bar{x}, \frac{1}{2}\right) P_{i}\left(x_{i}, p_{i}(x)\right)\right\} .
$$

Individuals who are below the median position $\left(p_{i}(x)<1 / 2\right)$ and below the average consumption $\left(x_{i}<\bar{x}\right)$ are always disadvantaged. Similarly, individuals who are above the median position $\left(p_{i}(x)>1 / 2\right)$ and above the average consumption $\left(x_{i}>\bar{x}\right)$ are always advantaged. However, individuals who are above (below) the median position but below (above) the average consumption may be disadvantaged (advantaged). Note that the highest income individual is always advantaged, the lowest income individual always disadvantaged.

We denote by $D^{\prime}(x)$ the subset of individuals who are in the bottom half of the consumption distribution:

$$
D^{\prime}(x)=\left\{i \in N \mid p_{i}(x)<\frac{1}{2}\right\}
$$

Using $D^{\prime}(x)$ instead of $D(x)$ as a subset of disadvantaged individuals, we define the following transfer axiom.

${ }^{30}$ The leximin social ordering with respect to personal consumption satisfies all axioms. 
Axiom 7. T': For any economy $E=\left(N, R_{N}\right)$, any allocations $x$ and $y$ in $\mathbb{R}^{n}$, any individuals $i \in D^{\prime}(x) \cap D^{\prime}(y), j \in A^{\prime}(x) \cap A^{\prime}(y)$ and any $\Delta>0$ :

$\left[y_{i}=x_{i}+\Delta, \quad y_{j}=x_{j}-\Delta, \quad x_{k}=y_{k} \forall k \neq i, j \quad\right.$ and $\left.\quad p_{k}(x)=p_{k}(y) \forall k \in N\right] \Rightarrow y R(E) x$.

As noted before, $D^{\prime}(x)$ may not coincide with $D(x)$ because some individuals may be above (below) the median position and still disadvantaged (advantaged). Therefore the corresponding requirements are not equivalent. However, imposing both Separability (or the stronger Separation) and Transfer to Disadvantaged, forces us to satisfy $\left(T^{\prime}\right)$.

Lemma 1. On $\mathcal{R}^{P}$, for $n \geq 3$, if a social ordering function satisfies Separability and Transfer to Disadvantaged, then it also satisfies $\left(\mathbf{T}^{\prime}\right)$.

The proof consists in using Separability to modify the average consumption so as to make Transfer to Disadvantaged applicable. Thanks to lemma 1, we can start our analysis from axiom ( $\left.\mathrm{T}^{\prime}\right)$, which is much easier to manipulate than Transfer to Disadvantaged on the domain of Positional Externalities.

\subsection{Characterization}

Any social ordering function which satisfies Strong Pareto, Unchanged Contour Independence, Separation and Transfer to Disadvantaged must be an egalitarian equivalent maximin social ordering function.

Theorem 2. On $\mathcal{R}^{P}$, if a social ordering function $R$ (.) satisfies Strong Pareto, Transfer to Disadvantaged, Unchanged Contour Independence, and Separation, then for all $E=\left(N, R_{N}\right)$ and $x, y$ in $\mathbb{R}^{n}$ :

$$
\min _{i \in N} u_{i}(x)>\min _{i \in N} u_{i}(y) \Rightarrow x P(E) y
$$

The proof follows the same general structure as in the model of average externalities. ${ }^{31}$ We first show that imposing Strong Pareto, Unchanged-Contour Independence and Separation forces us to go from a finite (Transfer to Disadvantaged) to an infinite aversion to inequality (Priority to Disadvantaged). The Priority property then implies that society should respect the opinions of disadvantaged individuals when they all agree about the comparisons between two allocations (Disadvantaged Unanmity). The final step consists in using Separation by adding individuals at both ends of the consumption distribution so as to reduce the gap in relative positions between the individuals of the initial economy, which allows to close the proof.

\section{Conclusion}

This paper studies the fair allocation of a one-dimensional and perfectly divisible good when individuals have other-regarding preferences. In contrast with the existing literature, mostly inspired by the Theory of Fair Allocation, we follow the approach of Fair Social Choice (Fleurbaey and Maniquet 2011 [17]), which aims at constructing complete orderings of all allocations. In two

\footnotetext{
${ }^{31}$ Note, however, that the argument used in each of these steps is quite different, which underlines the difficulty of obtaining a proof for more general domains.
} 
simple models of Average and Positional externalities, we characterize a class of social preferences which satisfy appealing efficiency, equity and consistency properties. These rankings require giving full priority to the worst-off individual, where an agent's welfare is measured in a particular constructed way. Our proofs rely on the geometry of the corresponding consumption spaces and do not generalize easily to any form of externalities. However, even in the general case, our social preferences do satisfy all proposed requirements on a broad domain of individual preferences, including some of the well known models of other-regarding preferences.

As made clear by the title of the paper, we choose here to follow the ideal of equality of welfare. Other ethical principles, capturing differing views about how to account for externalities in social welfare, may also be considered in the same model. One could argue in favor of a principle of anonymity, demanding that any allocation be socially indifferent to all its permutations, ${ }^{32}$ or a principle that rewards pro-social preferences. The investigation of such alternative requirements will be part of the research agenda on the topic. Future work could also involve extending the model to multi-dimensional goods, divisibles or indivisibles, as well as investigating the implications for public policy such as optimal income taxation.

\section{ProOFs}

$\mathbf{U}\left(x, R_{i}\right)$ denotes the upper contour set of $R_{i}$ at allocation $x$.

\subsection{General Setup}

\subsubsection{Proposition 1}

Proof. Existence.

Let $x$ be any allocation in $\mathbb{R}^{n}$. By continuity, the sets $A^{+}=\left\{\alpha \in \mathbb{R} \mid(\alpha, \ldots, \alpha) R_{i} x\right\}$ and $A^{-}=$ $\left\{\alpha \in \mathbb{R} \mid x R_{i}(\alpha, \ldots, \alpha)\right\}$ are closed. Furthermore, by Condition (II) in Reasonable Envy, there exists $-\infty<m<M<+\infty$ such that:

$$
\left(\max \left(M, x_{i}\right), \ldots, \max \left(M, x_{i}\right)\right) R_{i} x \quad \text { and } \quad x R_{i}\left(\min \left(m, x_{i}\right), \ldots, \min \left(m, x_{i}\right)\right) .
$$

Therefore, both $A^{+}$and $A^{-}$are non-empty. Since $R_{i}$ is complete, we also have $\mathbb{R} \subset A^{+} \cup A^{-}$, which then implies $A^{+} \cap A^{-} \neq \emptyset$ by the connectedness of $\mathbb{R}$ and the fact that $A^{+}$and $A^{-}$are closed and non-empty. We conclude that there exists $u_{i}(x) \in \mathbb{R}$ such that $x I_{i}\left(u_{i}(x), \ldots, u_{i}(x)\right)$.

Unicity.

Assume there exists $\lambda_{1}<\lambda_{2}$ such that both $x I_{i}\left(\lambda_{1}, \ldots, \lambda_{1}\right)$ and $x I_{i}\left(\lambda_{2}, \ldots, \lambda_{2}\right)$. Then, by Condition (I) in Reasonable Envy we get $\left(\lambda_{2}, \ldots, \lambda_{2}\right) P_{i}\left(\lambda_{1}, \ldots, \lambda_{1}\right)$, a contradiction.

Note that for any two allocations $x$ and $y$ in $\mathbb{R}^{n}$, Condition (I) in Reasonable Envy also implies

$$
x R_{i} y \quad \Leftrightarrow \quad\left(u_{i}(x), \ldots, u_{i}(x)\right) R_{i}\left(u_{i}(y), \ldots, u_{i}(y)\right) \quad \Leftrightarrow \quad u_{i}(x) \geq u_{i}(y) .
$$

The egalitarian equivalent provides a numerical representation of individual preferences.

\subsubsection{Proposition 2}

\footnotetext{
${ }^{32}$ Interestingly, it is possible to satisfy Strong Pareto, Transfer to Disadvantaged and Anonymity while exhibiting a strict aversion to inequality: $x R(E) y \Leftrightarrow \sum_{i \in N} \phi\left(x_{i}\right) \geq \sum_{i \in N} \phi\left(x_{i}\right)$, where $\phi($.$) is increasing, concave and such that$ $\max _{x, y \in \mathbb{R}} \frac{\phi^{\prime}(x)}{\phi^{\prime}(y)}<1+n \min _{i \in N}\left\{M R S_{i}(z)>0, z \in \mathbb{R}^{n}\right\}$.
} 


\section{RAFAEL TREIBICH}

Proof. Strong Pareto, Unchanged-Contour Independence and Separability follow immediatly from the fact that the egalitarian equivalent represents individual preferences and only depends on the individual's indifference curve at the considered allocation.

We show that it also satisfies Transfer to Disadvantaged. Let allocations $x$ and $y$ be as in the premise of the axiom. Then, since $i$ is disadvantaged, $(\bar{x}, \ldots, \bar{x}) P_{i} x I_{i}\left(u_{i}(x), \ldots, u_{i}(x)\right)$, so that $\bar{x}>u_{i}(x)$ by condition (I) in Reasonable Envy. Similarly, since $j$ is advantaged, $\bar{x} \leq u_{i}(x)$. Therefore, because $y P_{k} x$ for all $k \neq i$, we get $u_{i}(x) \leq u_{i}(y)<\bar{x} \leq u_{j}(x), u_{j}(y)$ and $u_{k}(x) \leq u_{k}(y)$. We conclude that $y$ dominates $x$ for the egalitarian equivalent leximin social ordering.

\subsection{Average Externalities}

For any allocation $x$ and any number $a \in \mathbb{R}$, we denote by $x_{i}\left(a, R_{i}\right)$ the quantity for which individual $i$ is indifferent between bundles $\left.\left(x_{i}\left(a, R_{i}\right)\right), a\right)$ and $\left(x_{i}, \bar{x}\right)$ :

$$
\left(x_{i}\left(a, R_{i}\right), a\right) I_{i}\left(x_{i}, \bar{x}\right) .
$$

$x_{i}\left(a, R_{i}\right)$ is always well defined on $\mathcal{R}^{A}$.

\subsubsection{Proposition 3}

Proof. Let $R($.$) be a social ordering function which satisfies Strong Pareto, Transfer to Disadvantaged,$ Unchanged Contour Independence and Separability. We show that it must then satisfy Priority to Disadvantaged.

Let $x$ and $y$ be such that:

$$
x_{i}<\bar{x} \leq x_{j}, \quad y_{i}<\bar{y} \leq y_{j}, \quad y P_{i} x, \quad x P_{j} y \quad \text { and } \quad x I_{k} y \forall k \neq i, j .
$$

$\mathbf{1}^{\text {st }}$ Case: $\bar{x}=\bar{y}$. Transfer to Disadvantaged applies directly.

$\mathbf{2}^{\text {nd }}$ Case: $\bar{x}<\bar{y}$. Consider allocation $z$ defined by:

$$
z_{i}=x_{i}\left(\bar{y}, R_{i}\right), \quad z_{k}=y_{k} \quad \forall k \neq i, j \quad \text { and } \quad z_{j}=n \bar{y}-\sum_{k \neq j} z_{k} .
$$

We have $\bar{z}=\bar{y}>\bar{x}$, so Reasonable Envy implies that $x$ can neither Pareto dominate nor be Pareto indifferent to $z$. Since $z I_{k} x$ for all $k \neq j$, we must necessarily have $z P_{j} x$. Thus, by Strong Pareto, $z P(E) x$. Furthermore, since $z_{i}<y_{i}<\bar{z}=\bar{y}<y_{j}<z_{j}$ and $z_{k}=y_{k}$ for all $k \neq i, j$, Transfer to Disadvantaged also implies y $P(E) z$. We conclude that y $P(E) x$.

$3^{\text {rd }}$ Case: $\bar{x}>\bar{y}$. Let $\varepsilon>0$ and define allocation $z$ by:

$$
z_{k}=x_{k}\left(\bar{x}+\varepsilon, R_{k}\right) \forall k \neq j \quad \text { and } \quad z_{j}=n(\bar{x}+\varepsilon)-\sum_{k \neq j} z_{k} .
$$

Note that $z I_{k} x$ for all $k \neq j$ and $z P_{j} x$ (by Reasonable Envy).

For any $\delta>0$, define allocation $z^{\delta}$ by:

$$
z_{i}^{\delta}=y_{i}-\delta, \quad z_{j}^{\delta}=y_{j}+\delta, \quad \text { and } \quad z_{k}^{\delta}=y_{k} \quad \forall k \neq i, j .
$$

For any $a \in\left[\bar{x}, u_{j}(z)\right]$ and $\delta>0$, define allocations $x^{\delta}(a)$ and $y^{\delta}(a)$ by:

$$
\begin{aligned}
& x_{i}^{\delta}(a)=z_{i}\left(a, R_{i}\right) \quad \text { and } \quad y_{i}^{\delta}(a)=z_{i}^{\delta}\left(a, R_{i}\right), \\
& y_{j}^{\delta}(a)=a \quad \text { and } \quad x_{j}^{\delta}(a)=z_{j}\left(a, R_{i}\right), \\
& x_{k}^{\delta}(a)=y_{k}^{a, \delta}=\frac{1}{n-2}\left(n a-x_{i}^{a, \delta}-x_{j}^{a, \delta}\right) \quad \forall k \neq i, j .
\end{aligned}
$$


By Continuity, the difference $y_{i}^{\delta}(\bar{x})-x_{i}^{\delta}(\bar{x})$ can be made arbitrarily small choosing $\delta$ close enough to $y_{i}-x\left(\bar{y}, R_{i}\right)$. Let $\delta^{*}$ be such that $y_{i}^{\delta^{*}}(\bar{x})-x_{i}^{\delta^{*}}(\bar{x})<z_{j}\left(\bar{x}, R_{i}\right)-\bar{x}=x_{j}^{\delta^{*}}(\bar{x})-y_{j}^{\delta^{*}}(\bar{x})$.

Let $x(a)=x^{\delta^{*}}(a)$ and $y(a)=y^{\delta^{*}}(a)$. We get:

$$
y_{i}(\bar{x})-x_{i}(\bar{x})<x_{j}(\bar{x})-y_{j}(\bar{x}) \quad \text { and } \quad y_{i}\left(u_{j}(z)\right)-x_{i}\left(u_{j}(z)\right)>0=x_{j}\left(u_{j}(z)\right)-y_{j}\left(u_{j}(z)\right) .
$$

By continuity, there must exist $\left.a^{*} \in\right] \bar{x}, u_{j}(z)\left[\operatorname{such}\right.$ that $x_{j}\left(a^{*}\right)-y_{j}\left(a^{*}\right)=y_{i}\left(a^{*}\right)-x_{i}\left(a^{*}\right)$.

Let $z^{\prime}=z^{\delta^{*}}\left(a^{*}\right), x^{\prime}=x\left(a^{*}\right)$ and $y^{\prime}=y\left(a^{*}\right)$. Note that by construction, $z I_{j} x^{\prime}$.

Construct preference relations $R_{j}^{\prime}$ and $R_{j}^{\prime \prime}$ such that:

$$
\begin{array}{r}
\mathbf{U}\left(z^{\prime}, R_{j}^{\prime \prime}\right)=\mathbf{U}\left(y^{\prime}, R_{j}^{\prime \prime}\right)=C o\left(\left\{w \in \mathbb{R}^{2}\left|\left(w_{j}-z_{j}^{\prime}\right) \geq(1+\varepsilon)\right| \bar{w}-\bar{y} \mid\right\},\right. \\
\left.\left\{w \in \mathbb{R}^{2}\left|\left(w_{j}-y_{j}^{\prime}\right) \geq(1+\varepsilon)\right| \bar{w}-\bar{y}^{\prime} \mid\right\}\right) \\
\mathbf{U}\left(z, R_{j}^{\prime \prime}\right)=\mathbf{U}\left(x^{\prime}, R_{j}^{\prime \prime}\right)=\operatorname{Co}\left(\left\{w \in \mathbb{R}^{2}\left|\left(w_{j}-x_{j}^{\prime}\right) \geq(1+\varepsilon)\right| \bar{w}-\bar{x}^{\prime} \mid\right\},\right. \\
\left.\left\{w \in \mathbb{R}^{2}\left|\left(w_{j}-z_{j}\right) \geq(1+\varepsilon)\right| \bar{w}-\bar{z} \mid\right\}\right)
\end{array}
$$

and

$$
\mathbf{U}\left(y, R_{j}^{\prime}\right)=\mathbf{U}\left(y, R_{j}^{\prime \prime}\right)=\mathbf{I}\left(y, R_{j}\right), \quad \mathbf{U}\left(x, R_{j}^{\prime}\right)=\mathbf{U}\left(x, R_{j}\right), \quad \mathbf{U}\left(z, R_{j}^{\prime}\right)=\mathbf{U}\left(z, R_{j}^{\prime \prime}\right),
$$

where $C o(A)$ denotes the convex hull of set A (see the example given in Figure 4). Choosing $\varepsilon>0$ small enough we get:

$$
\mathbf{U}\left(x, R_{j}^{\prime}\right) \subset \mathbf{U}\left(z, R_{j}^{\prime}\right) \quad \text { and } \quad \mathbf{U}\left(y, R_{j}^{\prime \prime}\right) \subset \mathbf{U}\left(z^{\prime}, R_{j}^{\prime \prime}\right) \subset \mathbf{U}\left(z, R_{j}^{\prime \prime}\right)
$$

so that both $R_{j}^{\prime}$ and $R_{j}^{\prime \prime}$ are well defined. Furthermore, we have:

$$
\begin{aligned}
& z^{\prime} I_{j}^{\prime \prime} y^{\prime}, \quad z I_{j}^{\prime \prime} x^{\prime}, \quad x I_{k} y, \quad x^{\prime} I_{k} y^{\prime} \quad \forall k \neq i, j, \\
& x_{j}^{\prime}-y_{j}^{\prime}=y_{i}^{\prime}-x_{i}^{\prime}, \quad x_{i}^{\prime}<y_{i}^{\prime}<\bar{y}^{\prime}=\bar{x}^{\prime} \leq y_{j}^{\prime}<x_{j}^{\prime} .
\end{aligned}
$$

Note that by construction both $R_{j}^{\prime}$ and $R_{j}^{\prime \prime}$ are in $\mathcal{R}^{A}$ and convex. Let $E^{\prime}=\left(N,\left(R_{j}^{\prime}, R_{-j}\right)\right)$ and $E^{\prime \prime}=\left(N,\left(R_{j}^{\prime \prime}, R_{-j}\right)\right)$ be the corresponding economies.

By contradiction, assume $x P(E) y$.

By Unchanged Contour Independence, $x P\left(E^{\prime}\right) y$.

By Strong Pareto, $z P\left(E^{\prime}\right) x$, so $z P\left(E^{\prime}\right) y$.

By Unchanged Contour Independence again, $z P\left(E^{\prime \prime}\right) y$.

By Transfer to Disadvantaged, $y R\left(E^{\prime \prime}\right) z^{\prime}$ so $z P\left(E^{\prime \prime}\right) z^{\prime}$.

Finally, by Separability, $x^{\prime} P\left(E^{\prime \prime}\right) y^{\prime}$.

However, by Transfer to Disadvantaged, we also have $y^{\prime} R\left(E^{\prime \prime}\right) x^{\prime}$, a contradiction. We conclude that $y R(E) x$.

Figure 4 illustrates the construction used in the proof for $n=3$.

\subsubsection{Theorem 1}

We introduce the following two properties.

\section{Axiom 8. Disadvantaged Unanimity}

For any economy $E=\left(N, R_{N}\right)$ and any allocations $x$ and $y$ in $\mathbb{R}^{n}$ such that $D(x)=D(y)$ : if $y P_{i} x$ for all $i$ in $D(x)=D(y)$, then $y(E) x$.

If an allocation is strictly preferred by all disadvantaged individuals while leaving all disadvantaged individuals disadvantaged and all advantaged individuals advantaged, then it is a strict social improvement. 


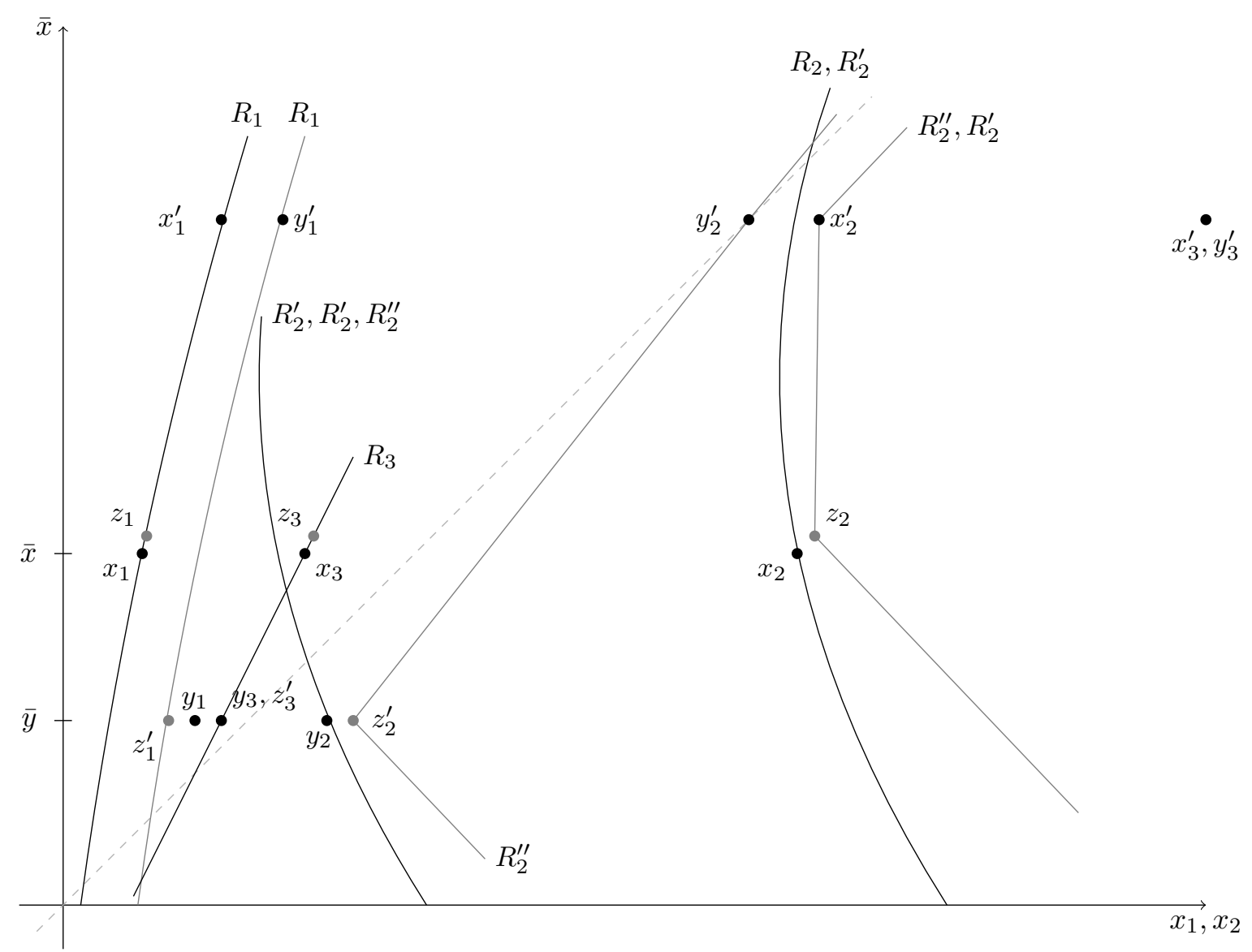

Figure 4. Allocation $y$ dominates allocation $x$ in the sense of Priority to Disadvantaged. By contradiction assume $x P(E) y$. By Unchanged Contour Independence, $x P\left(N, R_{N}^{\prime}\right) y$ (preferences remain unchanged at the corresponding indifference curves). By Strong Pareto $z P\left(N, R_{N}^{\prime}\right) x$, so $z P\left(N, R_{N}^{\prime}\right) y$. By Unchanged Contour Independence again, $z P\left(N, R_{N}^{\prime \prime}\right) y$. By Transfer to Disadvantaged, y $R\left(N, R_{N}^{\prime \prime}\right) z^{\prime}$ so $z P\left(N, R_{N}^{\prime \prime}\right) z^{\prime}$. Finally, by Separability we get $x^{\prime} P\left(N, R_{N}^{\prime \prime}\right) y^{\prime}$. However, by Transfer to Disadvantaged, we also have $y^{\prime} R\left(N, R_{N}^{\prime \prime}\right) x^{\prime}$, a contradiction. We conclude that $y R(E) x$.

\section{Axiom 9. Equal Split Selection}

For any economy $E=\left(N, R_{N}\right)$ and allocation $x \neq(\bar{x}, \ldots, \bar{x}) \in \mathbb{R}^{n}, \quad(\bar{x}, \ldots, \bar{x}) P(E) x$.

Any egalitarian allocation is strictly socially preferred to any other allocation of the same size. We start by showing the following proposition.

Proposition 4. On $\mathcal{R}^{A}$, Strong Pareto and Priority to Disadvantaged imply Disadvantaged Unanimity and Equal Split Selection.

Proof. Let $R($.$) be a social ordering function which satisfies Strong Pareto, Unchanged Contour Inde-$ pendence, Separability and Transfer to Disadvantaged. By Proposition 3, it also satisfies Priority to Disadvantaged.

Part 1: Disadvantaged Unanimity.

Let $x$ and $y$ be such that $y P_{i} x$ for any $i \in D(x)=D(y)$. Let 1 be one of the individuals with the lowest egalitarian equivalent at allocation $x: 1 \in \arg \min _{N} u_{i}(x)$. 
Step 1: Let $a>\max _{i \in N}\left\{u_{i}(x), u_{i}(y)\right\}$ and define allocation $z$ by:

$$
\begin{aligned}
& z_{1}=x_{1}\left(a, R_{1}\right) \\
& z_{i}=y_{i}\left(a, R_{i}\right) \quad \forall i \in D(x) \backslash\{1\} \\
& z_{i}=\frac{1}{n-d(x)}\left(n a-\sum_{j \in D(x)} z_{j}(a)\right) \quad \forall i \notin D(x),
\end{aligned}
$$

where $d(x)$ denotes $D(x)$ 's cardinal. At allocation $z, 1$ is indifferent to $x$ while all the other disadvantaged individuals are indifferent to $y$ (and thus better off than at $x$ ). Furthermore, because $a>\max _{N}\left(u_{i}(x), u_{i}(y)\right)$, all advantaged individuals at $x$ are still advantaged at $z$ and better off at $z$ than at $x$. By Strong Pareto, $z P(E) x$. Note that $\bar{z}>\max \{\bar{x}, \bar{y}\}$.

Step 2: Let $x^{0}=z$. For any integer $k>0$, define $A_{k}$ as the subset of advantaged individuals at allocation $x$ who are still better off at $x^{k-1}$ than at $y$ :

$$
A^{k}=\left\{i \in A(x) \mid x^{k-1} P_{i} y\right\}
$$

As long as $A^{k}$ is not empty, construct allocation $x^{k}$ as follows.

Let $i_{k}$ be any of the highest consumption individual in $A^{k}$ at allocation $x^{k-1}$ :

$$
i_{k} \in \underset{i \in A^{k}}{\arg \max } x_{i}^{k-1} \text {. }
$$

For any $a \geq \bar{y}$, define allocation $x^{k}(a, \varepsilon)$ by:

$$
\begin{aligned}
& x_{1}^{k}(a)=x_{1}^{k-1}\left(a, R_{1}\right)+\varepsilon, \\
& x_{j}^{k}(a)=x_{j}^{k-1}\left(a, R_{j}\right) \forall j \neq 1, i_{k}, \\
& x_{i_{k}}^{k}(a)=n a-\sum_{j \neq i_{k}} x_{j}^{k}(a),
\end{aligned}
$$

where $\varepsilon$ is chosen small enough so that $y P_{1} x^{k}(a)$ for all $a \geq \bar{y}, x^{k}\left(\bar{x}^{k-1}\right) P_{i_{k}} y$ and $x_{i_{k}}^{k}\left(\bar{x}^{k-1}\right)>\bar{x}^{k-1}$. By Continuity, since $y P_{i_{k}} x^{k}(\bar{y})$, there exists $\left.a^{1} \in\right] \bar{y}, \bar{x}^{k-1}\left[\right.$ such that $x^{k}\left(a^{1}\right) I_{i_{k}} y$.

$1^{\text {st }}$ Case: $x_{i_{k}}^{k}\left(a^{1}\right) \geq a^{1}$. Let $a^{*}=a^{1}$.

$2^{\text {nd }}$ Case: $x_{i_{k}}^{k}\left(a^{1}\right)<a^{1}$. Then, by Continuity, there exists $\left.a^{2} \in\right] a^{1}, \bar{x}^{k-1}\left[\right.$ such that $x_{i_{k}}^{k}\left(a^{2}\right)=a^{2}$ and $x^{k}\left(a^{2}\right) P_{i_{k}} y$. Let $a^{*}=a^{2}$.

Define $x^{k}=x^{k}\left(a^{*}\right)$. Note that we always have $\left.\bar{x}^{k} \in\right] \bar{y}, \bar{x}^{k-1}[$.

Step 3: We show that $A^{k}$ must necessarily become empty after a finite number of steps.

For any integer $k>0$, assume $A^{k} \neq \emptyset$. Then:

$1^{\text {st }}$ Case: $x^{k} I_{i_{k}} y$. This instance can occur at most $d(x)<n$ times.

$2^{\text {nd }}$ Case: $x_{i_{k}}^{k}=\bar{x}^{k}$ and $x^{k} P_{i_{k}} y$.

Let $m=\min _{i \in N} \inf _{x \in \mathbb{R}^{n}, \delta>0}\left\{\varepsilon \geq 0 \mid x-\delta e_{i}+\varepsilon \delta e_{-i} P_{i} x\right\}$. By Finite altruism, $m>0$. For any $j \neq i_{k}$ we have:

$$
m\left(x_{j}^{k}-x_{j}^{k-1}\right)<\left(\bar{x}^{k-1}-\bar{x}^{k}\right)
$$

Summing over $N$ we get,

$$
n m\left(\bar{x}^{k}-\bar{x}^{k-1}\right)<(n-1)\left(\bar{x}^{k-1}-\bar{x}^{k}\right)+m\left(\bar{x}^{k}-x_{i_{k}}^{k-1}\right),
$$

so that

$$
\bar{x}^{k-1}-\bar{x}^{k}>\left(x_{i_{k}}^{k-1}-\bar{x}^{k-1}\right)\left(n+\frac{n-1}{m}\right) .
$$


For any individual $i \notin A^{k}, y R_{i} x^{k-1}$, so by Reasonable Envy:

$$
x_{i}^{k-1}<y_{i}+\left(\bar{x}^{k-1}-\bar{y}\right),
$$

so that

$$
\sum_{i \notin A^{k}} x_{i}^{k-1}<\sum_{i \notin A^{k}} y_{i}+\left(n-a_{k}\right)\left(\bar{x}^{k-1}-\bar{y}\right)
$$

which then implies,

$$
\sum_{i \in A^{k}}\left(x_{i}^{k-1}-\bar{x}^{k-1}\right)>\sum_{i \in A^{k}}\left(y_{i}-\bar{y}\right)>a_{k} \min _{i \in D(y)}\left(y_{i}-\bar{y}\right) .
$$

Furthermore, since $i_{k}$ is the highest consumption individual in $A^{k}$ at allocation $x^{k-1}$,

$$
a_{k}\left(x_{i_{k}}^{k-1}-\bar{x}^{k-1}\right)>\sum_{i \in A^{k}}\left(x_{i}^{k-1}-\bar{x}^{k-1}\right)
$$

so that

$$
\left(x_{i_{k}}^{k-1}-\bar{x}^{k-1}\right)>\min _{i \in D(y)}\left(y_{i}-\bar{y}\right)>0 .
$$

Finally, we conclude that,

$$
\bar{x}^{k-1}-\bar{x}^{k}>\left(n+\frac{n-1}{m}\right) \min _{i \in D(y)}\left(y_{i}-\bar{y}\right)
$$

As long as $A^{k}$ is not empty, either some individual is brought to his indifference curve at $y$ ( $1^{\text {st }}$ Case) or the average $\bar{x}^{k}$ decreases by more than a fixed quantity ( $2^{\text {nd }}$ Case). However, because we must always have $\bar{x}^{k} \geq \bar{y}$, there necessarily exists some $k^{*}$ such that $A^{k^{*}}=\emptyset$. By Strong Pareto, we have y $P(E) x^{k^{*}}$. By transitivity, we conclude that $y P(E) x$.

Part 2: Equal Split Selection.

Equal Split Selection obtains from simple application of Disadvantaged Unanimity and Strong Pareto. By contradiction, assume there exists $x \in \mathbb{R}^{n}$ such that $x R(E)(\bar{x}, \ldots, \bar{x})$ and $x \neq(\bar{x}, \ldots, \bar{x})$. Let $b \in] \max _{i \in D(x)} u_{i}(x), \bar{x}[$, and define allocation $y$ by:

$$
\begin{aligned}
& y_{i}=b-\varepsilon \quad \forall i \in D(x), \\
& y_{i}=b+\frac{d}{n-d} \varepsilon \quad \forall i \notin D(x) .
\end{aligned}
$$

where $\varepsilon$ is chosen small enough so that $y P_{i} x \forall i \in D(x)$ and $(\bar{x}, \ldots, \bar{x}) P_{i} y \forall i \in N$. By Disadvantaged Unanimity, $y P(E) x$, so that $y P(E)(\bar{x}, \ldots, \bar{x})$. However, by Strong Pareto, we also have $(\bar{x}, \ldots, \bar{x}) P(E) y$, a contradiction.

Figure 5 illustrates the construction used in the proof of Lemma 2 (Disadvantaged Unanimity) for $n=3$.

We now give the proof for Theorem 1.

Proof. Let $R($.) be a social ordering function which satisfies Strong Pareto, Transfer to Disadvantaged, Unchanged-Contour Independence and Separability. By Proposition 3 and Lemma 2, it must also satisfy Disadvantaged Unanimity and Equal Split selection. Let $x$ and $y$ be any allocations such that $\min _{i \in N} u_{i}(x)>\min _{i \in N} u_{i}(y)$. We want to show that $x P(E) y$.

Let 1 be one of the individuals with the lowest egalitarian equivalent at allocation $y$. Let $b>\max _{N} u_{i}(x)$ and define allocation $z$ by:

$$
z_{1}=y_{1}\left(b, R_{1}\right) \quad z_{i}=\frac{1}{n-1}\left(n b-z_{1}\right) \quad \forall i>1
$$




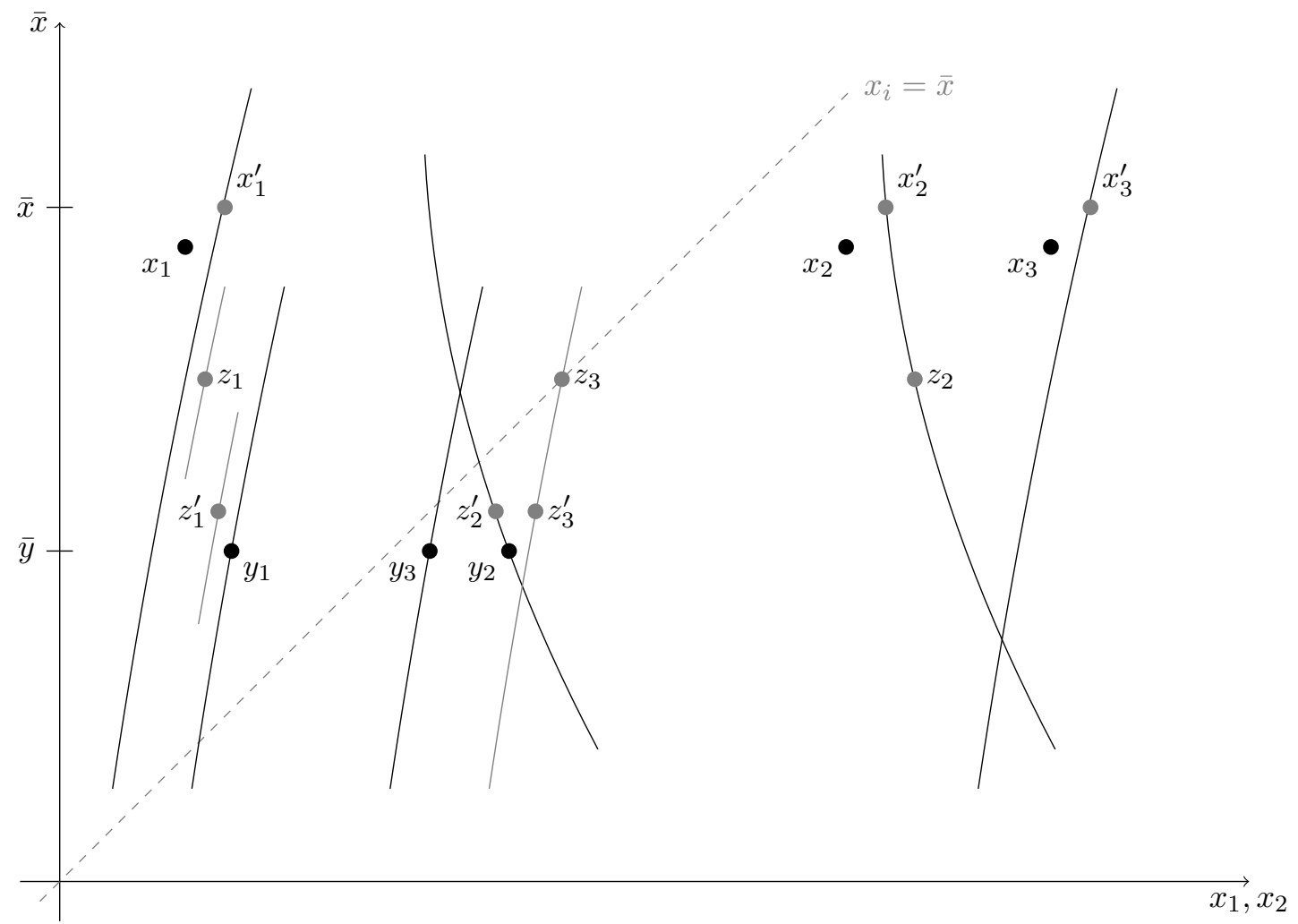

Figure 5. Allocation $x$ dominates allocations $y$ in the sense of Priority to Disadvantaged. The construction consists in progressively bringing all advantaged individuals to their indifference curve at allocation $y$, while making sure that none of them ever becomes disadvantaged. In this example individual 1 is the only disadvantaged individual in both $x$ and $y$. By Strong Pareto, $x^{\prime} P(E) x$. Then, by repeated application of Priority to Disadvantaged, $z R(E) x^{\prime}$, $z^{\prime} R(E) z$ and finally $y R(E) z^{\prime}$. By transitivity we conclude that $y R(E) x$.

Allocation $z$ is such that 1 remains indifferent to $y$ while all the other individuals are better off than at $y$ and advantaged. By Strong Pareto, $z P(E) y$.

Choose any $v \in] \min _{i \in N} u_{i}(y), \min _{i \in N} u_{i}(x)\left[\right.$ and define allocation $z^{\prime}$ by:

$$
z_{1}^{\prime}=v-(n-1) \delta, \quad z_{i}^{\prime}=v+\delta \quad \forall i>1
$$

where $\delta$ is chosen small enough so that $z^{\prime} P_{1} z$. Note that $\bar{z}^{\prime}=v$.

By Equal Split Transfer, $(v, \ldots, v) P(E) z^{\prime} .1$ is the only disadvantaged individual in both $z$ and $z^{\prime}$, and he prefers (strictly) $z^{\prime}$ to $z$. Therefore, by Disadvantaged Unanimity, $z^{\prime} R(E) z$. By transitivity, $(v, \ldots, v) R(E) y$. However, by Strong Pareto, we also have $x P(E)(v, \ldots, v)$. By transitivity, we conclude that $x P(E) y$.

To conclude the proof, we show the four axioms are independent by exhibiting social ordering functions which satisfy only three of these four axioms.

All axioms but Transfer to Disadvantaged: Take $R($.$) such that x R(E) y$ if and only if $\sum_{N} u_{i}(x) \geq$ $\sum_{N} u_{i}(y)$.

All axioms but Separability: Take $R($.$) such that x R(E) y$ if and only if $\bar{x} \geq \bar{y}$.

All axioms but Unchanged Contour Independence: Take $R($.$) such that:$ 


\section{RAFAEL TREIBICH}

(1) $x R(E) y$ if and only if $\bar{x} \geq \bar{y}$, when $R_{N}$ is such that $x R_{i} y \Leftrightarrow x_{i} \geq y_{i}$ for all $i \in N$.

(2) $x R(E) y$ if and only if $\min _{N} u_{i}(x) \geq \min _{N} u_{i}(y)$ for any other profile of preferences.

All axioms but Strong Pareto: Take $R($.$) such that x R(E) y$ if and only if $\max _{N} u_{i}(x) \leq \max _{N} u_{i}(y)$.

\subsubsection{Corrolary 1}

The proofs of Proposition 2 and Theorem 1 do not need any modification for $\mathcal{R}^{N A}$. For $\mathcal{R}^{P A}$, the construction used in proof of Proposition 2 can easily be adapted so as to use only preferences in $\mathcal{R}^{P A}$.

\subsection{Positional Externalities}

For any allocation $x \in \mathbb{R}^{n}$, any $a \in[0,1]$, and any preference relation $R_{i} \in \mathcal{R}^{P}$, we denote by $x_{i}\left(a, R_{i}\right)$ the quantity for which individual $i$ is indifferent between bundles $\left.\left(x_{i}\left(a, R_{i}\right)\right), a\right)$ and $\left(x_{i}, p_{i}(x)\right)$ :

$$
\left(x_{i}\left(a, R_{i}\right), a\right) I_{i}\left(x_{i}, p_{i}(x)\right)
$$

$x_{i}\left(a, R_{i}\right)$ is always well defined on $\mathcal{R}^{P}$.

\subsubsection{Lemma 1}

Proof. Assume allocations $x$ and $y$ are such that $y$ dominates $x$ in the sense of $\left(\mathrm{T}^{\prime}\right)$ :

$$
y_{i}=x_{i}+\Delta, \quad y_{j}=x_{j}-\Delta, \quad x_{k}=y_{k} \forall k \neq i, j \quad \text { and } \quad p_{k}(x)=p_{k}(y) \quad \forall k \in N
$$

We want to show that $y$ must be socially preferred to $x$.

$1^{\text {st }}$ Case: $i \in D(y), j \in A(y)$. Then, necessarily, $i \in D(x)$ and $j \in A(x)$. Transfer to Disadvantaged applies directly.

$2^{\text {nd }}$ Case: $i, j \in A(y)$. Then necessarily $j \in A(x)$ and $y_{i}>\bar{y}$. Note that $i$ cannot be the lowest income individual at allocation $x$. Define allocations $x^{\prime}$ and $y^{\prime}$ by:

$$
\begin{aligned}
& x_{k}^{\prime}=x_{k}, \quad y_{i}^{\prime}=y_{k}, \quad x_{j}^{\prime}=x_{j}, \quad y_{j}^{\prime}=y_{j}, \\
& x_{k}^{\prime}=y_{k}^{\prime}=x_{k} \text { if } x_{k} \geq x_{i}, \\
& x_{k}^{\prime}=y_{k}^{\prime}=x_{k}-\frac{\varepsilon}{p_{k}(x)} \text { if } x_{k}<x_{i},
\end{aligned}
$$

where $\varepsilon$ is chosen small enough so that $y_{i}^{\prime}<\overline{x^{\prime}}=\bar{y}^{\prime}<y_{j}^{\prime}$. Note that the relative order of all individuals is the same in allocations $x, y, x^{\prime}$ and $y^{\prime}$. Therefore, $i \in D\left(x^{\prime}\right) \cap D\left(y^{\prime}\right)$ and $j \in A\left(x^{\prime}\right) \cap A\left(y^{\prime}\right)$, so by Transfer to Disadvantaged $y^{\prime} P(E) x^{\prime}$. Finally, by Separability y $P(E) x$.

$3^{\text {rd }}$ Case: $i, j \in D(y)$. The proof follows in the same fashion as in previous case, using Separability to increase the average consumption (instead of decreasing it).

$4^{\text {th }}$ Case: $i \in A(y), j \in D(y)$. Impossible.

Using $D^{\prime}(x)$ instead of $D(x)$ as the set of disadvantaged individuals, we define the following variants of Priority to Disadvantaged and Disadvantaged Unanimity.

Axiom 10. $P$ ': For any economy $E=\left(N, R_{N}\right)$, any allocations $x$ and $y$ in $\mathbb{R}^{n}$, any individuals $i \in D^{\prime}(x) \cap D^{\prime}(y), j \in A^{\prime}(x) \cap A^{\prime}(y)$ and any $\Delta>0$ :

$$
\left[y P_{i} x, \quad x P_{j} y, \quad x_{k}=y_{k} \forall k \neq i, j \quad \text { and } \quad p_{k}(x)=p_{k}(y) \forall k \in N\right] \Rightarrow y R(E) x .
$$

Note that $\left(\mathrm{P}^{\prime}\right)$ only applies to pairs of allocations for which all relative positions remain unchanged (which therefore ensures all individuals besides $i$ and $j$ remain indifferent to the transfer).

Axiom 11. DU': For any economy $E=\left(N, R_{N}\right)$ and any allocations $x$ and $y$ in $\mathbb{R}^{n}$ such that $D^{\prime}(x)=$ $D^{\prime}(y)$ : if $y P_{i} x$ for all $i$ in $D^{\prime}(x)=D^{\prime}(y)$, then $y P(E) x$. 
7.3.2. Theorem 2. We start by showing the following two propositions.

Proposition 5. On $\mathcal{R}^{P}$, if a social ordering function satisfies Strong Pareto, Unchanged Contour Independence, Separability and $\boldsymbol{T}^{\prime}$ then it also satisfies $\boldsymbol{P}^{\prime}$.

Proof. Let $R($.$) be a social ordering function which satisfies Strong Pareto, Unchanged-Contour Inde-$ pendence, Separability and Transfer to Disadvantaged. Let $x$ and $y$ be any allocations in $\mathbb{R}^{n}$ such as in the premise of $\mathrm{P}$ '.

Wlog, we can assume that no individual shares his relative position at either $x$ or $y$ (otherwise, by Separability, just consider allocations $\tilde{x}$ and $\tilde{y}$, where the consumption of all but $i$ and $j$ is slightly modified, while remaining equal, so that all ties are broken). First define allocation $\hat{x}$ as follows:

$$
\begin{aligned}
& \hat{x}_{j}=x_{j}+N, \\
& \hat{x}_{k}=x_{k} \text { if } p_{k}(x)<p_{j}(x), \\
& \hat{x}_{k}=x_{k}+M \text { if } p_{k}(x)>p_{j}(x) .
\end{aligned}
$$

where $0<N<M$ are chosen big enough so that both $\hat{x}_{j}-y_{j} \geq y_{i}-\hat{x}_{i}$ and $\hat{x}_{k}>u_{j}(x)$ for any individual $k$ ranked above $j$.

$1^{\text {st }}$ Case: $p_{j}(x)>1 / 2$.

Let $l \in N$ be the (unique) individual who precedes $j$ in the consumption distribution at both $x$ and $y$. Construct allocations $x^{\prime}$ and $y^{\prime}$ such that:

$$
\begin{aligned}
& x_{k}^{\prime}=\hat{x}_{k} \quad \text { and } \quad y_{k}^{\prime}=y_{k} \quad \forall k \neq j, l, \\
& x_{j}^{\prime}=\hat{x}_{j}\left(p_{j}(x)-\frac{1}{n-1}, R_{j}\right), \quad y_{j}^{\prime}=y_{j}\left(p_{j}(y)-\frac{1}{n-1}, R_{j}\right) \\
& x_{l}^{\prime}=y_{l}^{\prime}=x_{j}^{\prime}+\varepsilon
\end{aligned}
$$

where $\varepsilon>0$ is chosen small enough so that the relative positions of all the other individuals remain unchanged. By construction, all individuals besides $l$ are indifferent between $x$ and $x^{\prime}$ and between $y$ and $y^{\prime}$, while $l$ is indifferent between $x$ and $y$ and between $x^{\prime}$ and $y^{\prime}$.

Define allocations $z$ and $z^{\prime}$ by:

$$
z_{i}=z_{i}^{\prime}=x_{i}+\delta, \quad z_{j}^{\prime}=x_{j}^{\prime}-\delta, \quad z_{j}=y_{j}+\left(y_{i}-z_{i}\right), \quad \text { and } \quad z_{k}=z_{k}^{\prime}=x_{k} \quad \forall k \neq i, j,
$$

where $\delta>0$ is chosen small enough so that $z_{i}<y_{i}$ and $z_{j}<z_{j}^{\prime}$. Note that all relative positions also remain unchanged at allocations $z$ and $z^{\prime}$.

Finally, construct preference relation $R_{j}^{\prime}$ and $R_{j}^{\prime \prime}$ such that $\mathbf{U}_{j}(x)=\mathbf{U}_{j}^{\prime}(x), \mathbf{U}_{j}(y)=\mathbf{U}_{j}^{\prime}(y)=\mathbf{U}_{j}^{\prime \prime}(y)$ and:

$$
\begin{gathered}
\mathbf{U}\left(z, R_{j}^{\prime \prime}\right)=\mathbf{U}\left(z^{\prime}, R_{j}^{\prime \prime}\right)=C o\left(\left\{(a, b) \in \mathbb{R}^{2} \mid a \geq z_{j}, b \geq p_{j}(z)-\varepsilon\left(a-z_{j}\right),\right\},\right. \\
\left.\left\{(a, b) \in \mathbb{R}^{2} \mid a \geq z_{j}^{\prime}, b \geq p_{j}\left(z^{\prime}\right)-\varepsilon\left(a-z_{j}^{\prime}\right)\right\}\right) \\
\mathbf{U}\left(\hat{x}, R_{j}^{\prime \prime}\right)=\mathbf{U}\left(x^{\prime}, R_{j}^{\prime \prime}\right)=C o\left(\left\{(a, b) \in \mathbb{R}^{2} \mid a \geq \hat{x}_{j}, b \geq p_{j}(\hat{x})-\varepsilon\left(a-\hat{x}_{j}\right),\right\},\right. \\
\left.\left\{(a, b) \in \mathbb{R}^{2} \mid a \geq x_{j}^{\prime}, b \geq p_{j}\left(x^{\prime}\right)-\varepsilon\left(a-x_{j}^{\prime}\right)\right\}\right)
\end{gathered}
$$

Let $E=\left(N, R_{N}\right), E^{\prime}=\left(N,\left\{R_{-j}, R_{j}^{\prime}\right\}\right)$ and $E^{\prime \prime}=\left(N,\left\{R_{-j}, R_{j}^{\prime \prime}\right\}\right)$ denote the corresponding economies.

By contradiction, assume $x R(E) y$.

By Unchanged Contour Independence, $x P\left(E^{\prime}\right) y$ because indifference curves of $R_{j}$ and $R_{j}^{\prime}$ coincide at both $x$ and $y$. 
By Strong Pareto, $\hat{x} P\left(E^{\prime}\right) x$, so $\hat{x} P\left(E^{\prime}\right) y$.

By Unchanged Contour Independence, $\hat{x} P\left(E^{\prime \prime}\right)$ y because indifference curves of $R_{j}^{\prime}$ and $R_{j}^{\prime \prime}$ coincide at both $\hat{x}$ and $y$.

By Separability, $x^{\prime} P\left(E^{\prime \prime}\right) y^{\prime}$.

By Transfer to Disadvantaged, $z^{\prime} P\left(E^{\prime \prime}\right) x^{\prime}$, so that $z^{\prime} P\left(E^{\prime \prime}\right) y^{\prime}$ (we know that $j$ is still advantaged at both $x^{\prime}$ and $y^{\prime}$ because $j$ is assumed to be strictly above the median position at both $x$ and $y$ ).

By Separability again, $z P\left(E^{\prime \prime}\right) y$.

However, by Transfer to Disadvantaged we also have y $R\left(E^{\prime \prime}\right) z$, a contradiction. We conclude that $y R(E) x$.

$2^{\text {nd }}$ Case: $p_{j}(x)=1 / 2$. In this case, the previous construction (moving someone ranked just below $j$ just above him) is not possible anymore because individual $j$ would be made disadvantaged, and we could not apply Transfer to Disadvantaged anymore. Instead, we need to take someone above $j$ and move him below. However, if $j$ cares too much about his relative position, leaving both $i$ and $j$ indifferent in the process may be unfeasible. An additional step is thus required.

First, define allocation $z$ from $y$ by transferring some small $\delta$ from $i$ to $j$ :

$$
z_{i}=y_{i}-\delta, \quad z_{j}=y_{j}+\delta, \quad z_{k}=y_{k} \forall k \neq i, j,
$$

where $\delta$ is chosen small enough so that $\hat{z}_{i}>x_{i}$. Then, define allocation $\hat{z}$ from $x$ by giving an additional $\varepsilon$ to individual $j$ :

$$
\hat{z}_{j}=x_{j}+\varepsilon, \quad \hat{z}=x_{k} \quad \forall k \neq j,
$$

where $\varepsilon>0$ is chosen small enough so that $p_{j}(\hat{z})=p_{j}(x)$.

Construct preference relations $R_{j}^{\prime}$ and $R_{j}^{\prime \prime}$ such that:

$$
\begin{aligned}
& \mathbf{U}\left(z, R_{j}^{\prime \prime}\right)=\left\{(a, b) \in \mathbb{R}^{2} \mid a \geq z_{j} \text { and }(a, b) R_{j} z\right\}, \\
& \mathbf{U}\left(\hat{z}, R_{j}^{\prime}\right)=\mathbf{U}\left(\hat{z}, R_{j}^{\prime \prime}\right)=\left\{(a, b) \in \mathbb{R}^{2} \mid a \geq \hat{z}_{j} \text { and }(a, b) R_{j} \hat{z}\right\},
\end{aligned}
$$

and

$$
\mathbf{U}\left(y, R_{j}^{\prime}\right)=\mathbf{U}\left(y, R_{j}^{\prime \prime}\right)=\mathbf{U}\left(y, R_{j}\right), \quad \mathbf{U}\left(x, R_{j}^{\prime}\right)=\mathbf{U}\left(x, R_{j}\right) \quad \text { and } \quad \mathbf{U}\left(y, R_{j}^{\prime}\right)=\mathbf{U}\left(y, R_{j}\right) .
$$

Let $E^{\prime}=\left(N,\left(R_{j}^{\prime}, R_{-j}\right)\right)$ and $E^{\prime \prime}=\left(N,\left(R_{j}^{\prime \prime}, R_{-j}\right)\right)$ be the corresponding economies. By contradiction assume $x P(E) y$.

By Strong Pareto, $\hat{z} P(E) x$, so $\hat{z} P(E) y$.

By Unchanged Contour Independence, $\hat{z} P\left(E^{\prime}\right) y$.

By Transfer to Disadvantaged, $y R\left(E^{\prime}\right) z$, so $\hat{z} P\left(E^{\prime}\right) z$.

By Unchanged Contour Independence, $\hat{z} P\left(E^{\prime \prime}\right) z$.

Following the same type of construction we used in the first case (this time moving the individual ranked just above $j$ just below him) for allocations $\hat{z}$ and $z$ in economy $E^{\prime \prime}$, we finally get to a contradiction. Note that such a contruction is necessarily possible in economy $E^{\prime \prime}$ (as opposed to economy $E$ ) because $j$ 's indifference curves at allocation $z$ and $\hat{z}$ are now vertical above the median line.

To conclude the proof, we check that the four axioms are necessary by exhibiting social ordering functions which satisfy only three of these four axioms.

All axioms but Transfer to Disadvantaged: Take $R($.$) such that x R(E) y$ if and only if $\sum_{N} u_{i}(x) \geq$ $\sum_{N} u_{i}(y)$.

All axioms but Separability: Take $R($.$) such that x R(E) y$ if and only if $\bar{x} \geq \bar{y}$.

All axioms but Unchanged Contour Independence: Take $R($.$) such that:$

(1) $x R y$ if and only if $\bar{x} \geq \bar{y}$, when $R_{N}$ is such that $x R_{i} y \Leftrightarrow x_{i} \geq y_{i}$ for all $i \in N$.

(2) $x R(E) y$ if and only if $\min _{N} u_{i}(x) \geq \min _{N} u_{i}(y)$ for any other profile of preferences. 
WELFARE EGALITARIANISM WITH OTHER-REGARDING PREFERENCES

All axioms but Strong Pareto: Take $R($.$) such that x R(E) y$ if and only if $\max _{N} u_{i}(x) \leq \max _{N} u_{i}(y)$.

Figure 6 illustrates the construction used in the proof for $n=3$.

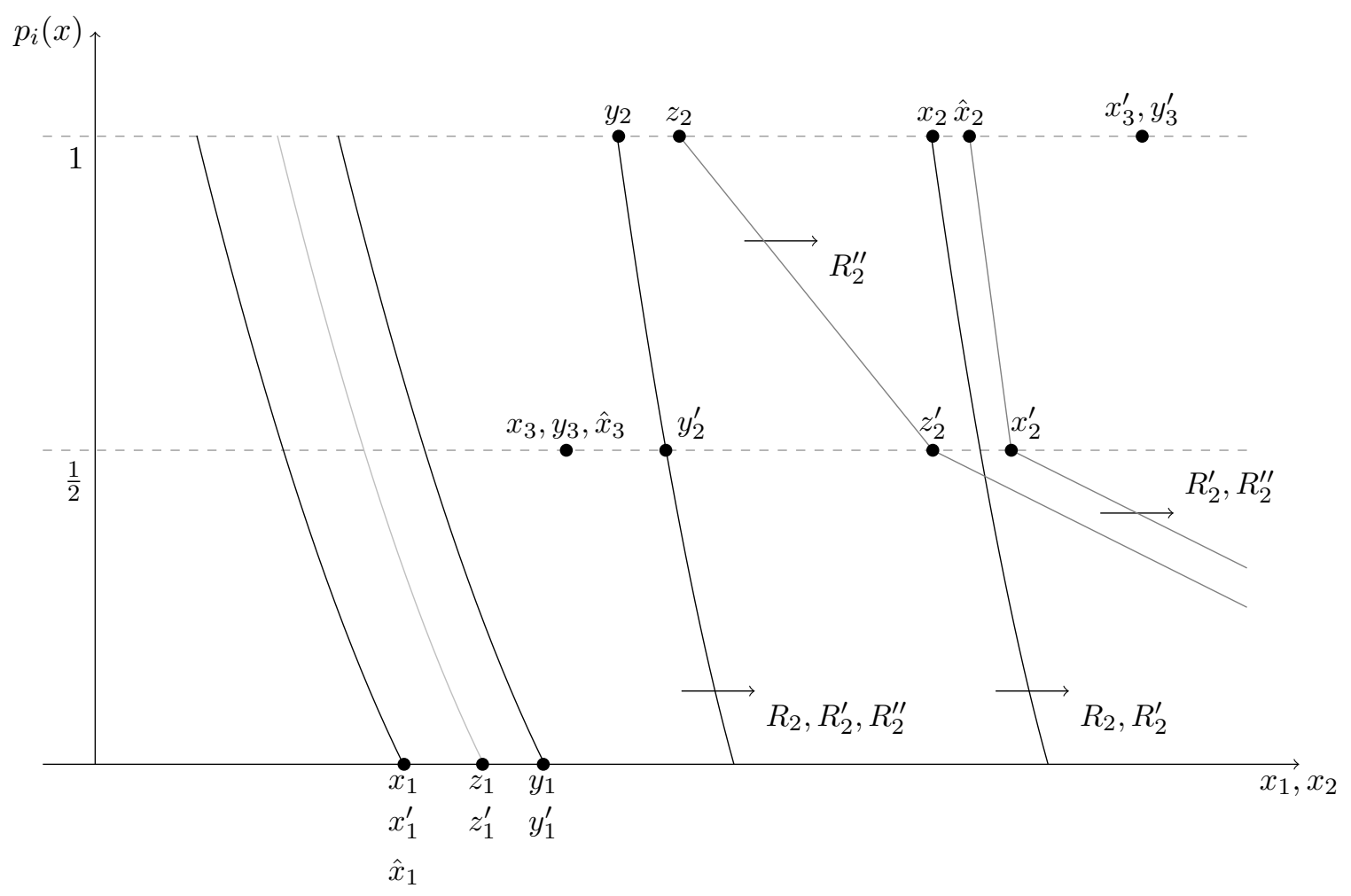

Figure 6. By contradiction, assume that $x P(E) y$. By Unchanged Contour Independence, $x P\left(E^{\prime}\right) y$ because indifference curves of $R_{2}$ and $R_{2}^{\prime}$ coincide at both $x$ and $y$. By Strong Pareto, $\hat{x} P\left(E^{\prime}\right) x P\left(E^{\prime}\right) y$. By Unchanged-Contour Independence, $\hat{x} P\left(E^{\prime \prime}\right) y$. By Separability, $x^{\prime} P\left(E^{\prime \prime}\right) y^{\prime}$. By $\left(\mathrm{T}^{\prime}\right), z^{\prime} P\left(E^{\prime \prime}\right) x^{\prime}$, so that $z^{\prime} P\left(E^{\prime}\right) y^{\prime}$. By Separability again, $z P\left(E^{\prime \prime}\right) y$. However, by $\left(\mathrm{T}^{\prime}\right)$ we also have $y R\left(E^{\prime \prime}\right) z$, a contradiction. We conclude that $y R(E) x$.

Proposition 6. On $\mathcal{R}^{P}$, if a social ordering function satisfies Strong Pareto and $\boldsymbol{P}^{\prime}$ then it also satisfies $\boldsymbol{D} \boldsymbol{U}^{\prime}$.

Proof. Let $R($.$) be a social ordering function which satisfies Strong Pareto and P'.$

Let $x$ and $y$ be such that $y P_{i} x$ for any $i \in D^{\prime}(x)=D^{\prime}(y)$. Wlog, assume $y_{1} \leq y_{2} \leq \cdots \leq y_{n}$. Let $d=\# D^{\prime}(x)$.

First define allocation $\hat{y}$ as follows:

$$
\hat{y}_{d}=y_{d}, \quad \hat{y}_{i}=y_{i} \text { if } y_{i} \neq y_{d} \quad \text { and } \quad \hat{y}_{i}=y_{i}-\varepsilon \text { if } i \neq d \text { and } y_{i}=y_{d},
$$

where $\varepsilon>0$ is chosen small enough so that all the individuals (besides $d$ ) for which $y_{i}=y_{d}$ still prefer $\hat{y}$ to $x$ and are still better ranked than all other disadvantaged individuals (besides $d$ ). By Strong Pareto, we have $y P(E) \hat{y}$. At allocation $\hat{y}, d$ is the only individual with the highest relative position among the disadvantaged individuals. Define allocation $z$ by:

$$
\begin{aligned}
z_{d} & =\hat{y}_{d}-\delta, \\
z_{i} & =\hat{y}_{i} \quad \forall i \in D^{\prime}(x) \backslash d, \\
z_{i} & =\max _{j \in N} u_{j}(x)+p_{i}(y) \quad \forall i \notin D^{\prime}(x),
\end{aligned}
$$




\section{RAFAEL TREIBICH}

where $\delta>0$ is chosen small enough so that $d$ is still the only highest ranked disadvantaged individual and $z P_{d} x$. Since $z P_{i} x$ for all $i \in N$, Strong Pareto implies $z P(E) x$. Note that all individuals share the same relative position at allocations $\hat{y}$ and $z$. Set $z^{0}=z$ and for any $k \in\{1, \ldots, n-d\}$ define:

$$
\begin{aligned}
& z_{d}^{k}=z_{d}+\frac{k}{n-d} \delta, \\
& z_{d+k}^{k}=y_{d+k}, \\
& z_{i}^{k}=z_{i} \quad \forall i \in N \neq d, d+k .
\end{aligned}
$$

Note that by construction, $z^{n-d}=\hat{y}$.

For any $k \in\{1, \ldots, n-d\}$ all relative positions coincide at allocations $z^{k}, z$ and $\hat{y}$, so that $z^{k} I_{i} z^{k-1}$ for any $i \neq d, d+k, d \in D^{\prime}\left(z^{k-1}\right) \cap D^{\prime}\left(z^{k}\right), d+k \in A^{\prime}\left(z^{k-1}\right) \cap A^{\prime}\left(z^{k}\right), z^{k} P_{d} z^{k-1}$ and $z^{k-1} P_{d+k} z^{k}$. By Priority to Disadvantaged, $z^{k} R(E) z^{k-1}$ for any $k \in\{1, \ldots, n-d\}$, so that $\hat{y}=z^{n-d} R(E) z$. By transitivity, we conclude that $y P(E) x$.

We now give the proof for Theorem 2

Proof. Let $R($.$) be a social ordering function which satisfies Strong Pareto, Transfer to Disadvantaged,$ Unchanged-Contour Independence, and Separation (and therefore Separability). By propositions 4 and 5 , it must also satisfy P' and DU'. Let $x$ and $y$ be any allocations such that $\min _{i \in N} u_{i}(y)>\min _{i \in N} u_{i}(x)$. We want to show that $y P(E) x$.

Let 1 be (one of) the individuals with the smallest egalitarian equivalent at allocation $x$. Define allocation $z$ by:

$$
z_{1}=x_{1}\left(0, R_{1}\right), \quad z_{i}=M \quad \forall i>1
$$

where $M$ is chosen large enough so that all the other individuals prefer (strictly) $z$ to $x$. Note that 1 is now the only disadvantaged individual at allocation $z$. Construct preference relation $\tilde{R}$ such that:

$$
U\left(\left(z_{i}, 1\right), \tilde{R}\right) \subset U\left(\left(z_{i}, 1\right), R_{i}\right) \forall i>1
$$

Let $E^{\prime}=\left(N,\left(R_{1}, \tilde{R}, \ldots, \tilde{R}\right)\right)$ be the corresponding economy. Choose any $\left.v \in\right] u_{1}(z), \min _{i \in N} u_{i}(y)[$. For any $r \in \mathbb{N}^{*}$, define allocations $z^{r}, v^{r}$ and $\hat{z}^{r}$ in $\mathbb{R}_{+}^{n+2 r}$ by:

$$
\begin{aligned}
& z_{1}^{r}=z_{1}\left(\frac{r}{n+2 r-1}, R_{1}\right), \quad z_{i}^{\prime}=z_{i}\left(\frac{3 r+n-1}{2(n+2 r-1)}, \tilde{R}\right) \quad \forall i \in N \backslash\{1\} \\
& v_{i}^{r}=v \quad \forall i \in N \\
& z_{i}^{r}=v_{i}^{r}=a<u_{1}(x) \quad \forall i \in\{n+1, \ldots, n+r\} \\
& z_{i}^{r}=v_{i}^{r}=b>\max _{i \in N} u_{i}(z) \quad \forall i \in\{n+r+1, \ldots, n+2 r\} \\
& \hat{z}_{1}^{r}=z_{1}^{r}+\varepsilon, \quad \hat{z}_{i}^{r}=z_{1}^{r}+2 \varepsilon \quad \forall i \in N \backslash\{1\} \\
& \hat{z}_{i}^{r}=z_{i}^{\prime} \quad \forall i \in\{n+1, \ldots, n+2 r\} .
\end{aligned}
$$

Taking both $\mathrm{r}$ large enough and $\varepsilon$ small enough we can always ensure that $v^{r} P_{i} \hat{z}^{r}$ for any $i \in N$. Let $r^{*}$ be such an integer and define the corresponding allocations $z^{\prime}=z^{r^{*}}, v^{\prime}=z^{r^{*}}$ and $\hat{z}=\hat{z}^{r^{*}}$.

Let $N^{\prime}=N \cup\{n+1, \ldots, n+2 r\}$, and $E^{\prime \prime}=\left(N^{\prime}, R_{N^{\prime}}^{\prime}\right)$ where the profile of preferences in $N^{\prime} \backslash N$ is chosen arbitrarily in $\mathcal{R}^{P}$.

By contradiction, assume $x P(E)(v, \ldots, v)$.

By Unchanged-Contour Independence, $x P\left(E^{\prime}\right)(v, \ldots, v)$.

By Strong Pareto, $z P\left(E^{\prime}\right) x$, so $z P\left(E^{\prime}\right)(v, \ldots, v)$.

By Separation, $z^{\prime} P\left(E^{\prime \prime}\right) v^{\prime}$.

By Disadvantaged Unanimity, $\hat{z} P\left(E^{\prime \prime}\right) z^{\prime}$, so that $\hat{z} P\left(E^{\prime}\right) v^{\prime}$.

However by Strong Pareto, $v^{\prime} P\left(E^{\prime \prime}\right) \hat{z}$, a contradiction. 
We conclude that $(v, \ldots, v) P(E) x$. Finally, by Strong Pareto we also have y $P(E)(v, \ldots, v)$, so that $y P(E) x$.

We show that the four axioms are indeed necessary.

All axioms but Transfer to Disadvantaged: Take $R($.$) such that x R(E) y$ if and only if $\sum_{N} u_{i}(x) \geq$ $\sum_{N} u_{i}(y)$.

All axioms but Separation: Take $R($.$) such that x R(E) y$ if and only if $x \geq^{\text {lex }} y$.

All axioms but Unchanged Contour Independence: Take $R($.$) such that:$

(1) $x R y$ if and only if $\bar{x} \geq \bar{y}$, when $R_{N}$ is such that $x R_{i} y \Leftrightarrow x_{i} \geq y_{i}$ for all $i \in N$.

(2) $x R(E) y$ if and only if $\min _{N} u_{i}(x) \geq \min _{N} u_{i}(y)$ for any other profile of preferences.

All axioms but Strong Pareto: Take $R($.$) such that x R(E) y$ if and only if $\max _{N} u_{i}(x) \leq \max _{N} u_{i}(y)$.

\section{REFERENCES}

[1] Aronsson T., Johansson-Stenman O., 2008. "When the Joneses' consumption hurts: Optimal public good provision and nonlinear income taxation", Journal of Public Economics, Vol. 92, pages 986-997.

[2] Bolton G.E., Ockenfels A., 2000. "ERC: a theory of equity, reciprocity, and competition", American Economic Review, Vol. 90(1), pages 166-193.

[3] Boskin M.J., Sheshinski E., 1978. "Optimal redistributive taxation when individual welfare depends upon relative income", Quarterly Journal of Economics, Vol. 92, pages 589-601.

[4] Charness G., Rabin M., 2002. "Understanding social preferences with simple tests", Quarterly Journal of Economics, Vol.117, pages 817-869.

[5] Clark A.E., Frijters P. and Shields M.A., 2008. "Relative income, happiness, and utility: An explanation for the Easterlin paradox and other puzzles", Journal of Economic Literature, Vol.46, pages 95144.

[6] Corneo G., Jeanne O., 1997. "Conspicuous Consumption, Snobbism and Conformism”, Journal of Public Economics, Vol. 66, pages 55-71.

[7] Cooper B., Garcia-Penalosa C. and Funk P., 2001. "Status Effects and Negative Utility Growth", The Economic Journal, Vol.111, pages 642-665.

[8] Decerf B., Van der Linden M., 2014. "Fair Social Orderings with Other-Regarding Preferences", Working Paper.

[9] Dufwenberg M., Heidhues P., Kirchsteiger G. and Riedel F., 2011. "General Equilibrium with Other-Regarding Preferences", Review of Economic Studies, Vol. 218, pages 613-639.

[10] Easterlin R., 1995. "Will Raising the Incomes of All Increase the Happiness of All?", Journal of Economic Behavior and Organization, Vol. 27.

[11] Fehr E., Schmidt K.M., 1999. "A theory of fairness, competition and cooperation", Quarterly Journal of Economics, Vol. 114, pages 817-868.

[12] Fleurbaey M., 2005. "The Pazner-Schmeidler Social Ordering: A Defense", Review of Economic Design, Vol. 9, pages $145-166$.

[13] Fleurbaey M., 2007. "Two criterias for Social Decisions", Journal of Economic Theory, Vol. 134, $421-447$.

[14] "The Importance of What People Care About", Politics, Philosophy and Economics, Vol. 11(4), pages 415-447.

[15] Fleurbaey M., Maniquet F., 2008. "Fair Social Orderings", Economic Theory, Vol. 34, pages 25-45.

[16] Fleurbaey M., Maniquet F., 2006. "Fair Income Tax", The Review of Economic Studies, Vol. 73, pages 55-83.

[17] Fleurbaey M., Maniquet F., 2011. "A Theory of Fairness and Social Welfare", Econometric Society Monograph, Cambridge University Press.

[18] Fleurbaey M., Schokkaert E., 2013. "Behavioral Welfare Economics and Redistribution", American Economic Journal: Microeconomics, Vol. 5(3), pages 180-205.

[19] Fleurbaey M., Tadenuma K., 2014. "Universal Social Orderings: An Integrated Theory of Policy Evaluation, InterSociety Comparisons, and Interpersonal Comparisons", The Review of Economic Studies, Vol. 81, pages 1071-1101.

[20] Fleurbaey M., Trannoy A., 2003. "The Impossibility of a Paretian Egalitarian", Social Choice and Welfare, Vol. 21, pages 243-263.

[21] Frank R. H., 1985. "Positional Externalities Cause Large and Preventable Welfare Losses", American Economic Review, Vol. 95(2), pages 137-141.

[22] Frank R. H., 2005. "Choosing the Right Pond", Oxford University Press.

[23] Gaertner W., 2006. "A Primer in Social Choice Theory", Oxford University Press, Oxford, UK. 


\section{RAFAEL TREIBICH}

[24] Goodin R., 1986. "Laundering Preferences", in J. Elster and A. Hylland (eds.), Foundations of Social Choice Theory, Cambridge University Press, pages 75-101.

[25] Heath, J., 2006. "Envy and Efficiency", Revue de philosophie Économique, Vol. 13.

[26] Ireland N.J., 2001. "Optimal Income Tax in the Presence of Status Effects", Journal of Public Economics, Vol. 81, pages 193-212.

[27] Kolm S.C., 1995. "The Economics of Social Sentiments: the Case of Envy", The Japanese Economic Review, Vol.46, pages $63-87$.

[28] Maniquet F., 2008. "Social Orderings for the Assignment of Indivisible Objects", Journal of Economic Theory, Vol. 143, No. 1, pages 199-215.

[29] Maniquet F., Sprumont Y., 2004. "Fair Production and Allocation of an Excludable Nonrival Good", Econometrica, Vol. 72, No. 2, pages 627-640.

[30] Maniquet F., Sprumont Y., 2005. "Welfare egalitarianism in non-rival environments", Journal of Economic Theory, Vol. 120, pages 155-174.

[31] Moulin H., 1992. "Welfare Bounds in the Cooperative Production Problem" Games and Economic Behavior, Vol. 4, pages 373-401.

[32] Nieto J., 1991. "A Note on Egalitarian and Efficient Allocations with Externalities in Consumption" Journal of Public Economics, Vol. 46, pages 261-266.

[33] Nogushi M., Zame W., 2006. "Competitive Markets with Externalities". Theoretical Economics, Vol. 1, pages 143166.

[34] Pazner E., Schmeidler D., 1978. "Egalitarian Equivalent Allocations: a New Concept of Economic Equity", Quarterly Journal of Economics, Vol. 92, pages 671-687.

[35] Persson M., 1995. "Why are Taxes So High in Egalitarian Societies?" Scandinavian Journal of Economics, Vol. 97, pages 569-580.

[36] Piacquadio P., 2014. "Ranking Intergenerational Allocations in Risky Environments " Working Paper.

[37] Pigou A.C., 1912. "Wealth and Welfare" London: Macmillan.

[38] Sen, A., 1970. "Collective Choice and Social Welfare", San Francisco: Holden Day.

[39] Sen, A., 1970. "The Impossibility of a Paretian Liberal", Journal of Political Economy, Vol. 78, pages 152-157.

[40] Sobel J., 2005. "Interdependent Preferences and Reciprocity", Journal of Economic Literature, Vol. 43, pages 392:436.

[41] Thomson W., 2010. "Fair Allocation Rules", in K. Arrow, A.K. Sen, and K. Suzumura (eds.), Handbook of Social Choice and Welfare Volume II . Amsterdam, New York:North-Holland.

[42] Valletta G., 2009. "A Fair Solution to the Compensation Problem", Social Choice and Welfare, Vol. 32, No. 3, pages 455-478.

[43] Varian H., 1974. "Efficiency, equity and envy", Journal of Economic Theory, Vol. 9, pages 63-91.

[44] Veblen T., 1899. "The theory of the leisure class", MacMillan, New York.

[45] Velez R., 2014. "Fairness with Externalities", Working Paper.

[46] Villar A., 1988. "On the Existence of Pareto Optimal Allocations when Individual Welfare Depends on Relative Consumption", Journal of Public Economics, Vol. 36, pages 387-397. 